\title{
MAY 101932
}

U. S. DEPARTMENT OF COMMERCE BUREAU OF STANDARDS

\section{WOOD POLES FOR OVERHEAD ELECTRICAL LINES}

HANDBOOK, BUREAU OF STANDARDS, No. 16

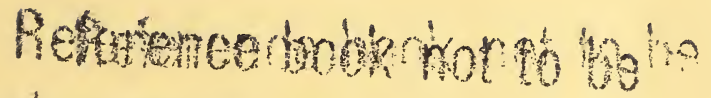

Intentwom the linary. 



\section{U. S. DEPARTMENT OF COMMERCE \\ R. P. LAMONT, Secretary \\ BUREAU OF STANDARDS \\ GEORGE K. BURGESS, Director}

HANDBOOK, BUREAU OF STANDARDS, No. 16

\section{WOOD POLES FOR \\ OVERHEAD ELECTRICAL \\ LINES}

[December 17, 1931]

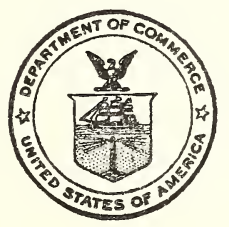

UNITED STATES

GOVERNMENT PRINTING OFFICE

WASHINGTON : 1932

For sale by the Superintendent of Documents, Washington, D. C. - Price 10 cents 



\section{CONTENTS}

Page

Introduction $\ldots \ldots \ldots \ldots \ldots$

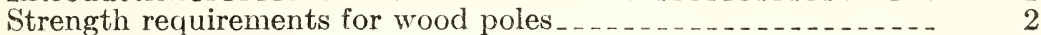

Table 19. Ultimate fiber stresses of wood poles

Table 20. Allowable fiber stresses for wood poles under vertical and transverse loading

Revised tables' for Appendix F of the National Electrical Safety Code-wood poles

Moments of resistance of poles

Transverse loads on poles

Vertical loads on poles

Longitudinal loads on poles

Depreciation of wood poles.

Allowable number of wires on a given pole without side guys.

Table 83A. Resisting moments for poles of southern yellow pine_

Table 83B. Resisting moments for poles of chestnut

Table 83C. Resisting moments for poles of western red cedar - -

Table 84. Resisting moments for poles of cypress ............

Table 85. Resisting moments for poles of northern white cedar and redwood

Table $87 \mathrm{~A}$. Ratio of ultimate fiber stresses to allowable fiber

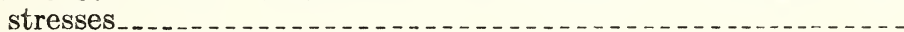

Table $87 \mathrm{~B}$. Moments due to wind pressure on poles. 



\section{WOOD POLES FOR OVERHEAD ELECTRICAL LINES}

\section{INTRODUCTION}

The American Standards Association, under date of November 28, 1930, approved the following standard values for ultimate fiber stresses of wood poles as used in electrical line construction.

Northern white cedar, 3,600 lbs. per sq. in.

Western red cedar, 5,600 lbs. per sq. in.

Chestnut, 6,000 lbs. per sq. in.

Southern yellow pine (creosoted), 7,400 lbs. per sq. in.

The employment of these standard values in designing lines to meet strength requirements is provided for in the National Electrical Safety Code. (See rule 261 A4(c).)

The value approved by the American Standards Association for northern white cedar is the same as that already recognized in the National Electrical Safety Code. The values for the three other woods will supersede those previously in use. (Dense yellow pine, 6,500 ; chestnut and western cedar, 5,000.)

The application of these values under the requirements of the code will introduce changes in Tables 19 and 20 of the code. The new standards also require certain changes in the tables of Appendix F, Wood Poles.

To make these changes available the requirements dealing: with wood poles and parts of Appendix $\mathrm{F}$ are reprinted here, incorporating the changes due to the new standard values of ultimate fiber stress.

The information in Appendix $\mathrm{F}$ has been augmented to include tables and a nomogram from which the transverse bending moment caused by the wind pressure on the projected area of the pole may be readily obtained. 


\section{STRENGTH REQUIREMENTS FOR WOOD POLES}

(Being the requirements of the National Electrical Safety Code revised in accordance with American Standard for Ultimate Fiber Stresses of Wood Poles, ASA 05a-1930.)

261. Grades A, B, and C Construction.

\section{A. Poles and Towers.}

4. Wood Poles.

Wood poles shall be of such material and dimensions as to meet the following requirements. Where guys are used see rule $261, \mathrm{C}$.

(a) TRANSverse Strength.-Wood poles shall withstand the transverse and vertical loads assumed in rule $254, \mathrm{~A}$ and $\mathrm{B}$, without exceeding at the ground line for unguyed poles, or at the point of guy attachment for guyed poles, the appropriate allowable fiber stresses given in Table 20.

(b) LONGITUdiNAL STRENGTH.-

Grades $A$ and $B$.- The longitudinal strength of wood poles shall be maintained at all times so that ther will withstand the longitudinal loading specified in rule $254, \mathrm{C}$, without exceeding at the ground line for unguyed poles, or at the point of guy attachment for guyed poles, the appropriate ultimate fiber stresses given in Table 19.

Grade C.-No longitudinal strength requirements except at dead ends.

(c) Ultimate FIBER STREss.-Difierent kinds of wood poles are considered as having the ultimate fiber stresses given in Table 19. These ultimate fiber stresses are given so as to identify different kinds of pole timbers with the ultimate fiber stresses appearing at the heads of the columns in Table 20.

Table 19-Ultimate fiber stresses of wood poles

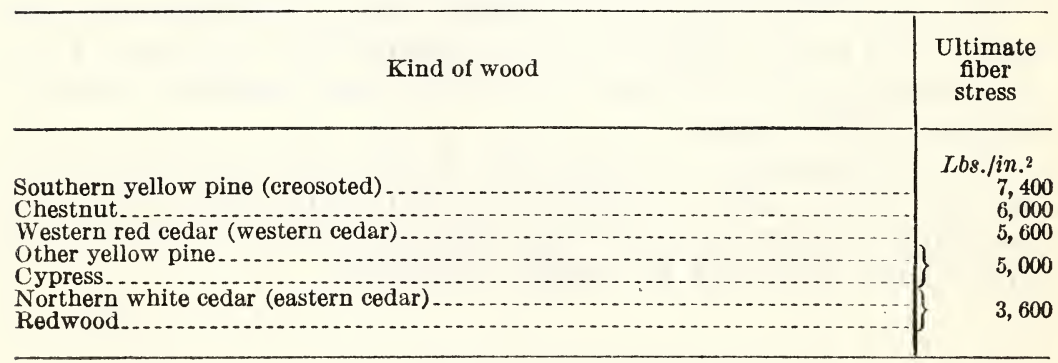


(d) TREATED POLEs.-The use of treated poles is not required. However, under certain circumstances Table 20 permits higher allowable stresses for treated than for untreated poles. Treated poles are poles meeting the following: requirements:

(1) Preservatives.- The preservatives used shall be coaltar creosote or other preservative equally satisfactory with regard to electrical resistance, retention of the preservative within the timber, and efficacy as to preservation. In the case of poles which are butt treated only, the electrical resistance of the preservative may be disregarded.

(2) Full-Length treatment.-Pine and other timber subject to rapid decay above ground shall be treated full length by a pressure process or some other equally effective method.

(3) Butt tREATMENT.-Cedar, chestnut, and other timber not subject to rapid decay above ground shall be treated by any process which will produce impregnation of most of the sapwood from at least 2 feet below the ground line to at least 1 foot above the ground line. In the case of treatments which require perforation, no method shall be used which results in perforation to the cross section required at replacement.

(e) ALLOWABLE FIBER STRESSES.-The allowable fiber stresses to be used in computing the strength of treated and untreated poles to withstand vertical and transverse loads are given in Table 20. 


\begin{tabular}{|c|c|c|c|c|c|c|c|c|}
\hline 屯 & \multirow{5}{*}{ 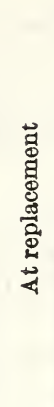 } & \multirow{5}{*}{ 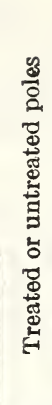 } & \multirow{5}{*}{ 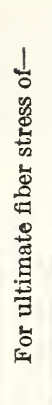 } & $\begin{array}{l}8 \\
\text { \&. } \\
\text { n }\end{array}$ & 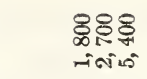 & & $\begin{array}{l}\text { ్ㅇ요요 } \\
\text {-ivis } \\
\text {-ivis }\end{array}$ & $\begin{array}{l}\text { 엄잉ㅇ } \\
\text { जimis }\end{array}$ \\
\hline 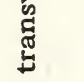 & & & & 8 & 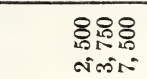 & & 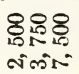 & $\begin{array}{l}888 \\
88: 5 \\
\text { mint }\end{array}$ \\
\hline ర్జ & & & & 8 & 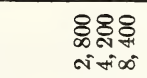 & & 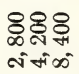 & $\begin{array}{l}\text { 융유 } \\
\text { mis } \\
\text { m. }\end{array}$ \\
\hline . & & & & $\begin{array}{l}8 \\
8 \\
0\end{array}$ & 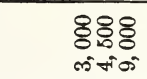 & & 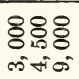 & 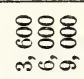 \\
\hline 岂 & & & & 원 & $\begin{array}{l}8.88 \\
8.00 \\
\text { m. }\end{array}$ & & $\begin{array}{l}808 \\
8.00 \\
\text { m.20 }\end{array}$ & $\begin{array}{l}\text { 육유 } \\
\text { fi゙= }\end{array}$ \\
\hline త్త & & & $\begin{array}{l}\frac{1}{0} \\
0 \\
8\end{array}$ & $\begin{array}{l}8 \\
8 \\
\text { s. }\end{array}$ & 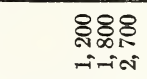 & & 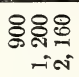 & 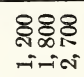 \\
\hline 8 & & 递 & 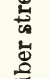 & 8 & 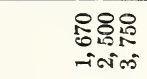 & & 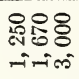 & $\begin{array}{l}\text { 융요요 } \\
\text { inim }\end{array}$ \\
\hline ڤ્ఝ & & $\begin{array}{l}\text { 苞 } \\
\text { 壳 }\end{array}$ & 弯 & $\begin{array}{l}8 \\
0 \\
0\end{array}$ & 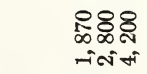 & & 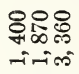 & $\begin{array}{l}\text { 용ㅇ } \\
\infty \\
\text {-ivi- }\end{array}$ \\
\hline . & 串 & & 竎 & $\begin{array}{l}8 \\
0 \\
0\end{array}$ & $\begin{array}{l}88 \% \\
880 \\
\text { Nint }\end{array}$ & & $\begin{array}{l}8808 \\
808 \\
\text { - }\end{array}$ & 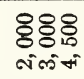 \\
\hline 营 & $\begin{array}{l}\frac{\pi}{62} \\
\Xi \\
0\end{array}$ & & $\perp$ & $\begin{array}{l}8 \\
8 \\
\text { के }\end{array}$ & 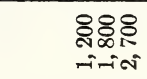 & & $\begin{array}{l}\text { ్ㅗㅇㅛ } \\
\text {-i-iv }\end{array}$ & $\begin{array}{l}\text { 안요 } \\
\text { rivim }\end{array}$ \\
\hline ప్రి & $\overrightarrow{3}$ & 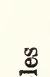 & 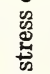 & 8 & 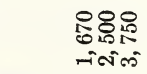 & & $\begin{array}{l}\text { 융오 } \\
0.00 \\
\text { rivin }\end{array}$ & $\begin{array}{l}8880 \\
880 \\
\text { ज. }\end{array}$ \\
\hline 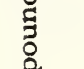 & & 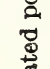 & : & $\begin{array}{l}8 \\
0 \\
15\end{array}$ & 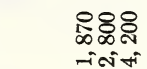 & & $\begin{array}{l}\text { 요요 } \\
\text { - } \\
\text { rivit }\end{array}$ & 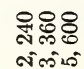 \\
\hline.$\Xi$ & & E & $\stackrel{g}{3}$ & $\begin{array}{l}8 \\
0 \\
0\end{array}$ & 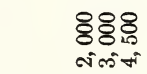 & & $\begin{array}{l}8888 \\
880 \\
\text { जin- }\end{array}$ & $\begin{array}{l}8880 \\
780 \\
\text { Nimo }\end{array}$ \\
\hline 岂 & & & o & \& & 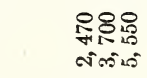 & & 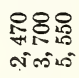 & $\begin{array}{l}\text { 영영 } \\
\text { जitin }\end{array}$ \\
\hline 崩 & & & & & 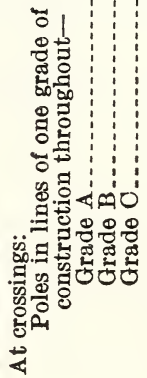 & 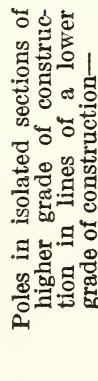 & 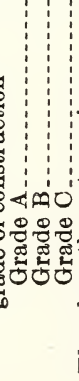 & 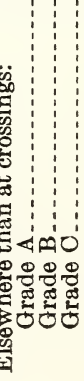 \\
\hline
\end{tabular}


( $f$ ) FREEDOM FROM DEFECTS.-Wood poles shall be selected timber free from observable defects that would decrease their strength and durability.

(g) MINIMUM POLE SIZEs.-Wood poles shall have nominal top diameters not less than the following:

Table 21.-Minimum top diameters for wood poles

\begin{tabular}{|c|c|c|c|}
\hline \multirow{2}{*}{ Grade of construction } & \multicolumn{3}{|c|}{$\begin{array}{l}\text { Minimum top diameters } \\
\text { for different loading } \\
\text { districts }\end{array}$} \\
\hline & $\begin{array}{c}\text { Heavy } \\
\text { (H) }\end{array}$ & $\underset{(\mathrm{M})}{\text { Medium }}$ & $\begin{array}{l}\text { Light } \\
\text { (L) }\end{array}$ \\
\hline $\begin{array}{l}\mathrm{A} \\
\mathrm{B}\end{array}$ & \begin{tabular}{|r} 
Inches \\
7 \\
6 \\
6
\end{tabular} & $\begin{array}{r}\text { Inches } \\
7 \\
6 \\
6\end{array}$ & $\begin{array}{r}\text { Inches } \\
6 \\
6 \\
6\end{array}$ \\
\hline
\end{tabular}

(h) SPLICED POLES.-Spliced poles shall not be used at crossings, conflicts, or joint-use sections requiring grade $\mathrm{A}$, $\mathrm{B}$, or $\mathrm{C}$ construction.

\section{REVISED TABLES FOR APPENDIX F OF NATIONAL ELECTRICAL SAFETY CODE-WOOD POLES}

\section{MOMENTS OF RESISTANCE OF POLES}

The resisting moments of wood poles of various groundline circumferences are given in the accompanying tables for each value of allowable fiber stress recognized in Table 20, for poles when installed. The tables are applicable to all woods having the recognized ultimate fiber stresses mentioned in the titles. Table $83 \mathrm{~A}$ applies especially to southern yellow pine (creosoted); Table $83 \mathrm{~B}$ applies especially to chestnut; Table $83 \mathrm{C}$ applies especially to western red cedar; Table 84 applies especially to cypress; and Table 85 to northern white cedar and redwood.

Southern yellow pine should not be used for supporting structures unless first given a preservative treatment, as otherwise the rapid deterioration will require early replacement. 
The resisting moment of a pole is expressed by the wellknown structural formula

where

$$
M=\frac{f I}{y}
$$

$M=$ moment.

$f=$ allowable fiber stress.

$\frac{I}{y}=$ section modulus.

For poles with circular cross section, this formula may be expressed in terms of the allowable fiber stress and circumference as follows:

where

$$
M=0.0002638 f G^{3}
$$

$M=$ moment in pound-feet.

$f=$ allowable fiber stress in pounds per square inch.

$G=$ circumference of the pole at ground line in inches.

While the ground-line section may not be the most stressed section in poles with considerable taper, it is so regarded here. Since the wood usually deteriorates most rapidly at this point, it is here that sufficient strength must be provided.

The last column in each of the Tables $83 \mathrm{~A}, 83 \mathrm{~B}, 83 \mathrm{C}, 84$, and 85 is for fiber stress 50 per cent greater than the ultimate. This value is included because, in construction of grade $\mathrm{C}$, poles are permitted to deteriorate to such a point, before replacement is called for, that the factor of safety is only two-thirds. This factor is based, however, upon an assumed loading which in some localities is experienced only at rare intervals, and by some individual poles perhaps never. Such a condition does not, therefore, indicate failure. 
Table 83A.-Resisting moments for poles of woods having an ultimate fiber stress of 7,400 pounds per square inch (southern yellow pine, creosoted)

\begin{tabular}{|c|c|c|c|c|c|c|c|}
\hline \multirow{2}{*}{$\begin{array}{l}\text { Circumference at } \\
\text { ground line } \\
\text { (inches) }\end{array}$} & \multicolumn{7}{|c|}{ Resisting moments for fiber stress of (pounds per square inch) } \\
\hline & 2,470 & 2,960 & 3,700 & 4,440 & 5,550 & 7,400 & 11,100 \\
\hline 20 - & $\begin{array}{c}L b .-f t . \\
5,200\end{array}$ & $\begin{array}{l}L b .-f t . \\
\quad 6,250\end{array}$ & $\begin{array}{c}L b .-f t . \\
7,800\end{array}$ & $\begin{array}{l}L b .-f t . \\
\quad 9,350\end{array}$ & $\begin{array}{l}L b .-f t . \\
11,700\end{array}$ & $\begin{array}{c}L b .-f t . \\
15,600\end{array}$ & $\begin{array}{l}L b .-f t . \\
\quad 23,450\end{array}$ \\
\hline $\begin{array}{l}24= \\
25-\end{array}$ & $\begin{array}{r}6,050 \\
6,950 \\
7,950 \\
9,000 \\
10,200\end{array}$ & $\begin{array}{r}7,250 \\
8,300 \\
9,500 \\
10,800 \\
12,200\end{array}$ & $\begin{array}{r}9,050 \\
10,400 \\
11,900 \\
13,500 \\
15,250\end{array}$ & $\begin{array}{l}10,850 \\
12,450 \\
14,250 \\
16,200 \\
18,300\end{array}$ & $\begin{array}{l}13,550 \\
15,600 \\
17,800 \\
20,250 \\
22,900\end{array}$ & $\begin{array}{l}18,100 \\
20,800 \\
23,750 \\
27,000 \\
30,500\end{array}$ & $\begin{array}{l}27,100 \\
31,200 \\
35,650 \\
40,500 \\
45,750\end{array}$ \\
\hline $\begin{array}{l}26 . \\
27 . \\
28 \\
29 . \\
30 .\end{array}$ & $\begin{array}{l}11,450 \\
12,850 \\
14,300 \\
15,900 \\
17,600\end{array}$ & $\begin{array}{l}13,700 \\
15,350 \\
17,150 \\
19,050 \\
21,100\end{array}$ & $\begin{array}{l}17,150 \\
19,200 \\
21,450 \\
23,800 \\
26,350\end{array}$ & $\begin{array}{l}20,600 \\
23,050 \\
25,700 \\
28,550 \\
31,600\end{array}$ & $\begin{array}{l}25,750 \\
28,800 \\
32,150 \\
35,700 \\
39,550\end{array}$ & $\begin{array}{l}34,300 \\
38,400 \\
42,850 \\
47,600 \\
52,700\end{array}$ & $\begin{array}{l}51,450 \\
57,650 \\
64,300 \\
71,400 \\
79,050\end{array}$ \\
\hline $\begin{array}{l}31- \\
32- \\
33- \\
34- \\
35\end{array}$ & $\begin{array}{l}19,400 \\
21,350 \\
23,400 \\
25,600 \\
27,950\end{array}$ & $\begin{array}{l}23,250 \\
25,600 \\
28,050 \\
30,700 \\
33,500\end{array}$ & $\begin{array}{l}29,100 \\
32,000 \\
35,100 \\
38,350 \\
41,850\end{array}$ & $\begin{array}{l}34,900 \\
38,400 \\
42,100 \\
46,050 \\
50,200\end{array}$ & $\begin{array}{l}43,600 \\
48,000 \\
52,600 \\
57,550 \\
62,750\end{array}$ & $\begin{array}{l}58,150 \\
63,950 \\
70,150 \\
76,750 \\
83,700\end{array}$ & $\begin{array}{r}87,250 \\
95,950 \\
105,250 \\
115,100 \\
125,550\end{array}$ \\
\hline $\begin{array}{l}36 . \\
37- \\
38- \\
39- \\
40-\end{array}$ & $\begin{array}{l}30,400 \\
33,000 \\
35,750 \\
38,650 \\
41,700\end{array}$ & $\begin{array}{l}36,450 \\
39,550 \\
42,850 \\
46,300 \\
49,950\end{array}$ & $\begin{array}{l}45,550 \\
49,450 \\
53,550 \\
57,900 \\
62,450\end{array}$ & $\begin{array}{l}54,650 \\
59,350 \\
64,250 \\
69,500 \\
74,950\end{array}$ & $\begin{array}{l}68,300 \\
74,150 \\
80,350 \\
86,850 \\
93,700\end{array}$ & $\begin{array}{r}91,100 \\
98,900 \\
107,100 \\
115,800 \\
124,950\end{array}$ & $\begin{array}{l}136,600 \\
148,300 \\
160,700 \\
173,700 \\
187,400\end{array}$ \\
\hline $\begin{array}{l}41 . \\
42- \\
43 . \\
44 . \\
45 .\end{array}$ & $\begin{array}{l}44,900 \\
48,250 \\
51,800 \\
55,500 \\
59,400\end{array}$ & $\begin{array}{l}53,800 \\
57,850 \\
62,100 \\
66,500 \\
71,150\end{array}$ & $\begin{array}{l}67,250 \\
72,300 \\
77,600 \\
83,150 \\
88,950\end{array}$ & $\begin{array}{r}80,750 \\
86,800 \\
93,100 \\
99,750 \\
106,750\end{array}$ & $\begin{array}{l}100,900 \\
108,450 \\
116,400 \\
124,700 \\
133,400\end{array}$ & $\begin{array}{l}134,550 \\
144,650 \\
155,200 \\
166,300 \\
177,900\end{array}$ & $\begin{array}{l}201,800 \\
216,950 \\
232,800 \\
249,450 \\
266,850\end{array}$ \\
\hline $\begin{array}{l}46 . \\
47 .- \\
48 . \\
49- \\
50 .\end{array}$ & $\begin{array}{l}63,400 \\
67,650 \\
72,050 \\
76,650 \\
81,450\end{array}$ & $\begin{array}{l}76,000 \\
81,050 \\
86,350 \\
91,850 \\
97,600\end{array}$ & $\begin{array}{r}95,000 \\
101,350 \\
107,950 \\
114,850 \\
122,000\end{array}$ & $\begin{array}{l}114,000 \\
121,600 \\
129,550 \\
137,800 \\
146,400\end{array}$ & $\begin{array}{l}142,500 \\
152,000 \\
161,900 \\
172,250 \\
183,000\end{array}$ & $\begin{array}{l}190,000 \\
202,650 \\
215,900 \\
229,650 \\
244,000\end{array}$ & $\begin{array}{l}285,000 \\
304,000 \\
323,850 \\
344,500 \\
366,000\end{array}$ \\
\hline $\begin{array}{l}51 . \\
52 . \\
53- \\
54 . \\
55 .\end{array}$ & $\begin{array}{r}86,450 \\
91,600 \\
97,000 \\
102,600 \\
108,400\end{array}$ & $\begin{array}{l}103,600 \\
109,800 \\
116,250 \\
122,950 \\
129,900\end{array}$ & $\begin{array}{l}129,500 \\
137,250 \\
145,300 \\
153,700 \\
162,400\end{array}$ & $\begin{array}{l}155,350 \\
164,700 \\
174,400 \\
184450 \\
194,850\end{array}$ & $\begin{array}{l}194,200 \\
205,850 \\
217,950 \\
230,550 \\
243,600\end{array}$ & $\begin{array}{l}258,950 \\
274,500 \\
290,650 \\
307,400 \\
324,800\end{array}$ & $\begin{array}{l}388,450 \\
411,750 \\
435,950 \\
461,100 \\
487,200\end{array}$ \\
\hline $\begin{array}{l}56 \ldots \\
57-. \\
58 \ldots \\
59 \ldots \\
60 \ldots\end{array}$ & $\begin{array}{l}114,450 \\
120,650 \\
127,150 \\
133,800 \\
140,750\end{array}$ & $\begin{array}{l}137,150 \\
144,600 \\
152,350 \\
160,350 \\
168,650\end{array}$ & $\begin{array}{l}171,400 \\
180,750 \\
190,450 \\
200,450 \\
210,850\end{array}$ & $\begin{array}{l}205,700 \\
216,900 \\
228,550 \\
240,550 \\
253,000\end{array}$ & $\begin{array}{l}257,100 \\
271,150 \\
285,650 \\
300,700 \\
316,250\end{array}$ & $\begin{array}{l}342,800 \\
361,500 \\
380,900 \\
400,900 \\
421,650\end{array}$ & $\begin{array}{l}514,250 \\
542,300 \\
571,300 \\
601,400 \\
632,500\end{array}$ \\
\hline $\begin{array}{l}61 \ldots \\
62 \ldots \\
63 \ldots \\
65 \\
65 \\
\ldots\end{array}$ & $\begin{array}{l}147,900 \\
155,300 \\
162,950 \\
170,800 \\
178,950\end{array}$ & $\begin{array}{l}177,250 \\
186,100 \\
195,250 \\
204,700 \\
214,450\end{array}$ & $\begin{array}{l}221,550 \\
232,600 \\
244,050 \\
255,850 \\
268,050\end{array}$ & $\begin{array}{l}265,850 \\
279,150 \\
292,850 \\
307,050 \\
321,650\end{array}$ & $\begin{array}{l}332,300 \\
348,950 \\
366,100 \\
383,800 \\
402,100\end{array}$ & $\begin{array}{l}443,100 \\
465,250 \\
488,100 \\
511,750 \\
536,100\end{array}$ & $\begin{array}{l}664,650 \\
697,850 \\
732,200 \\
767,600 \\
804,150\end{array}$ \\
\hline
\end{tabular}


Table 83A.- Resisting moments for poles of woods having an ultimate fiber stress of 7,400 pounds per square inch (southern yellow pine, creosoted)-Continued

\begin{tabular}{|c|c|c|c|c|c|c|c|}
\hline \multirow{2}{*}{$\begin{array}{l}\text { Circamference at } \\
\text { ground lines } \\
\text { (inches) }\end{array}$} & \multicolumn{7}{|c|}{ Resisting moments for fiber stress of (pounds per square inch) } \\
\hline & 2,470 & 2,960 & 3,700 & 4,440 & 5,550 & 7,400 & 11,100 \\
\hline 1 & $\begin{array}{l}L b .-f t . \\
187,350 \\
195,950 \\
204,900 \\
214,050 \\
223,500\end{array}$ & $\begin{array}{l}L b .-f t . \\
224,500 \\
234,850 \\
245,500 \\
256,500 \\
267,850\end{array}$ & $\begin{array}{l}L b .-f t . \\
280,600 \\
293.550 \\
306,900 \\
320,650 \\
334,800\end{array}$ & $\begin{array}{l}L b .-f t . \\
336,750 \\
352,300 \\
368,300 \\
384,750 \\
401,750\end{array}$ & $\begin{array}{l}L b .-f t . \\
420,900 \\
440,350 \\
460,350 \\
480,950 \\
502,200\end{array}$ & $\begin{array}{l}L b .-f t . \\
561,250 \\
587,150 \\
613,800 \\
641,300 \\
669,600\end{array}$ & $\begin{array}{r}\text { Lb.-ft. } \\
841,850 \\
880,700 \\
920,700 \\
961,950 \\
1,004,350\end{array}$ \\
\hline 1 & $\begin{array}{l}233,200 \\
243,200 \\
253,500 \\
264,050 \\
274,900\end{array}$ & $\begin{array}{l}279,450 \\
291,450 \\
303,750 \\
316,400 \\
329,400\end{array}$ & & & $\begin{array}{l}524,000 \\
546,450 \\
569,550 \\
593,300 \\
617,650\end{array}$ & $\begin{array}{l}698,700 \\
728,600 \\
759,400 \\
791,050 \\
823,550\end{array}$ & $\begin{array}{l}1,048,050 \\
1,092,950 \\
1,139,100 \\
1,186,550 \\
1,235,350\end{array}$ \\
\hline $\begin{array}{l}78 \\
79 \\
80\end{array}$ & $\begin{array}{l}286,050 \\
297,450 \\
309,200 \\
321,250 \\
333,600\end{array}$ & $\begin{array}{l}342,750 \\
356,500 \\
370,550 \\
385,000 \\
399,800\end{array}$ & $\begin{array}{l}428,450 \\
445,600 \\
463,200 \\
481,250 \\
499,750\end{array}$ & $\begin{array}{l}514,150 \\
534,700 \\
555,850 \\
577,500 \\
599,700\end{array}$ & $\begin{array}{l}642,700 \\
668,400 \\
694,800 \\
721,850 \\
749,600\end{array}$ & $\begin{array}{l}856,950 \\
891,200 \\
926,400 \\
962,450 \\
999,500\end{array}$ & $\begin{array}{l}1,285,400 \\
1,336,800 \\
1,389,550 \\
1,443,700 \\
1,499,250\end{array}$ \\
\hline
\end{tabular}

Table 83B.-Resisting moments for poles of woods having an ultimate fiber stress of 6,000 pounds per square inch (chestnut)

\begin{tabular}{|c|c|c|c|c|c|c|c|c|}
\hline \multirow{2}{*}{$\begin{array}{l}\text { Circumference at } \\
\text { ground line } \\
\text { (inches) }\end{array}$} & \multicolumn{8}{|c|}{ Resisting moments for fiber stress of (pounds per square inch) } \\
\hline & 1,500 & 2,000 & 2,400 & 3,000 & 3,600 & 4,500 & 6,000 & 9,000 \\
\hline 20. & $\begin{array}{l}L b .-f t . \\
3,150\end{array}$ & $\begin{array}{c}L b .-f t . \\
4,200\end{array}$ & $\begin{array}{r}L b .-f t . \\
5,050\end{array}$ & $\begin{array}{c}L b .-f t . \\
6,350\end{array}$ & $\begin{array}{c}L b .-f t . \\
7,600\end{array}$ & $\begin{array}{c}L b .-f t . \\
9,500\end{array}$ & $\begin{array}{l}L b .-f t . \\
12,650\end{array}$ & $\begin{array}{l}L b .-f t . \\
19,000\end{array}$ \\
\hline $\begin{array}{l}21 \ldots . . \\
22 \\
23 . . \\
24 .- \\
25 . .\end{array}$ & $\begin{array}{l}3,650 \\
4,200 \\
4,800 \\
5,450 \\
6,200\end{array}$ & $\begin{array}{l}4,900 \\
5,700 \\
6,400 \\
7,300 \\
8,250\end{array}$ & $\begin{array}{l}5,850 \\
6,850 \\
7,700 \\
8,750 \\
9,900\end{array}$ & $\begin{array}{r}7,350 \\
8,550 \\
9,650 \\
10,950 \\
12,350\end{array}$ & $\begin{array}{r}8,800 \\
10,200 \\
11,550 \\
13,150 \\
14,850\end{array}$ & $\begin{array}{l}11,000 \\
12,750 \\
14,450 \\
16,400 \\
18,550\end{array}$ & $\begin{array}{l}14,650 \\
16,950 \\
19,250 \\
21,900 \\
24,750\end{array}$ & $\begin{array}{l}22,000 \\
25,400 \\
28,900 \\
32,800 \\
37,100\end{array}$ \\
\hline $\begin{array}{l}26 .- \\
27-- \\
28 .- \\
29- \\
30 .\end{array}$ & $\begin{array}{r}6,950 \\
7,800 \\
8,700 \\
9,650 \\
10,700\end{array}$ & $\begin{array}{r}9,250 \\
10,400 \\
11,600 \\
12,850 \\
14,250\end{array}$ & $\begin{array}{l}11,150 \\
12,450 \\
13,900 \\
15,450 \\
17,100\end{array}$ & $\begin{array}{l}13,900 \\
15,600 \\
17,350 \\
19,300 \\
21,350\end{array}$ & $\begin{array}{l}16,700 \\
18,700 \\
20,850 \\
23,150 \\
25,650\end{array}$ & $\begin{array}{l}20,850 \\
23,350 \\
26,050 \\
28,950 \\
32,050\end{array}$ & $\begin{array}{l}27,800 \\
31,150 \\
34,750 \\
38,600 \\
42,750\end{array}$ & $\begin{array}{l}41,750 \\
46,750 \\
52,100 \\
57,900 \\
64,100\end{array}$ \\
\hline $\begin{array}{l}31 . \\
32 \\
33 \\
34 \\
35 \\
\text {. }\end{array}$ & $\begin{array}{l}11,800 \\
12,950 \\
14,200 \\
15,550 \\
16,950\end{array}$ & $\begin{array}{l}15,700 \\
17,300 \\
18,950 \\
20,750 \\
22,600\end{array}$ & $\begin{array}{l}18,850 \\
20,750 \\
22,750 \\
24,900 \\
27,150\end{array}$ & $\begin{array}{l}23,600 \\
25,950 \\
28,450 \\
31,100 \\
33,950\end{array}$ & $\begin{array}{l}28,300 \\
31,100 \\
34,150 \\
37,350 \\
40,700\end{array}$ & $\begin{array}{l}35,350 \\
38,900 \\
42,650 \\
46,650 \\
50,900\end{array}$ & $\begin{array}{l}47,150 \\
51,850 \\
56,900 \\
62,200 \\
67,850\end{array}$ & $\begin{array}{r}70,750 \\
77,800 \\
85,300 \\
93,300 \\
101,800\end{array}$ \\
\hline
\end{tabular}


Table 83B.-Resisting moments for poles of woods having an ultimate fiber stress of 6,000 pounds per square inch (chestnut)-Continued

\begin{tabular}{|c|c|c|c|c|c|c|c|c|}
\hline \multirow{2}{*}{$\begin{array}{l}\text { Circumference at } \\
\text { ground line } \\
\text { (inches) }\end{array}$} & \multicolumn{8}{|c|}{ Resisting moments for fiber stress of (pounds per square inch) } \\
\hline & 1,500 & 2,000 & 2,400 & 3,000 & 3,600 & 4,500 & 6,000 & 9,000 \\
\hline $\begin{array}{l}36 \ldots \ldots \\
38 \\
38 \ldots \ldots \\
98 \ldots \ldots \\
0\end{array}$ & $\begin{array}{l}L b .-f t . \\
18,450 \\
20,050 \\
21,700 \\
23,450 \\
25,300\end{array}$ & $\begin{array}{l}L b .-f t . \\
24,600 \\
26,700 \\
28,950 \\
31,300 \\
33,750\end{array}$ & $\begin{array}{c}L b .-f t . \\
29,550 \\
32,050 \\
34,750 \\
37,550 \\
40,500\end{array}$ & $\begin{array}{l}\text { Lb. } f t . \\
36,900 \\
40,100 \\
43,450 \\
46,950 \\
50,650\end{array}$ & $\begin{array}{l}\text { Lb.-ft. } \\
44,300 \\
48,100 \\
52,100 \\
56,350 \\
60,800\end{array}$ & $\begin{array}{l}L b .-f t . \\
55,400 \\
60,150 \\
65,150 \\
70,400 \\
75,950\end{array}$ & $\begin{array}{r}\text { Lb.-ft. } \\
73,850 \\
80,150 \\
86,850 \\
93,900 \\
101,300\end{array}$ & $\begin{array}{l}\text { Lb.-ft. } \\
110,750 \\
120,250 \\
130,300 \\
140,850 \\
151,950\end{array}$ \\
\hline ( & $\begin{array}{l}27,250 \\
29,300 \\
31,450 \\
33,700 \\
36,050\end{array}$ & $\begin{array}{l}36,350 \\
39,100 \\
41,950 \\
44,950 \\
48,100\end{array}$ & $\begin{array}{l}43,650 \\
46,900 \\
50,350 \\
53,950 \\
57,700\end{array}$ & $\begin{array}{l}54,550 \\
58,650 \\
62,900 \\
67,400 \\
72,100\end{array}$ & $\begin{array}{l}65,450 \\
70,350 \\
75,500 \\
80,900 \\
86,550\end{array}$ & $\begin{array}{r}81,800 \\
87,950 \\
94,400 \\
101,100 \\
108,150\end{array}$ & $\begin{array}{l}109,100 \\
117,250 \\
125,850 \\
134,850 \\
144,250\end{array}$ & $\begin{array}{l}163,650 \\
175,900 \\
188,750 \\
202,250 \\
216,350\end{array}$ \\
\hline 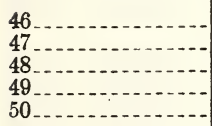 & $\begin{array}{l}38,500 \\
41,100 \\
43,750 \\
46,550 \\
49,450\end{array}$ & $\begin{array}{l}51,350 \\
54,800 \\
58,350 \\
62,050 \\
65,950\end{array}$ & $\begin{array}{l}61,650 \\
65,750 \\
70,000 \\
74,500 \\
79,150\end{array}$ & $\begin{array}{l}77,050 \\
82,150 \\
87,500 \\
93,100 \\
98,950\end{array}$ & $\begin{array}{r}92,450 \\
98,600 \\
105,050 \\
111,750 \\
118,700\end{array}$ & $\begin{array}{l}115,550 \\
123,250 \\
131,300 \\
139,650 \\
148,400\end{array}$ & $\begin{array}{l}154,050 \\
164,350 \\
175,050 \\
186,200 \\
197,850\end{array}$ & $\begin{array}{l}231,100 \\
246,500 \\
262,550 \\
279,300 \\
296,800\end{array}$ \\
\hline$-x$ & $\begin{array}{l}52,500 \\
55,650 \\
58,900 \\
62,300 \\
65,850\end{array}$ & $\begin{array}{l}70,000 \\
74,200 \\
78,550 \\
83,100 \\
87,800\end{array}$ & $\begin{array}{r}84,000 \\
89,000 \\
94,250 \\
99,700 \\
105,350\end{array}$ & $\begin{array}{l}105,000 \\
111,300 \\
117,800 \\
124,600 \\
131,650\end{array}$ & $\begin{array}{l}126,000 \\
133,550 \\
141,400 \\
149,550 \\
158,000\end{array}$ & $\begin{array}{l}157,450 \\
166,900 \\
176,750 \\
186,950 \\
197,500\end{array}$ & $\begin{array}{l}209,950 \\
222,550 \\
235,650 \\
249,250 \\
263,350\end{array}$ & $\begin{array}{l}314,950 \\
333,850 \\
353,450 \\
373,850 \\
395,000\end{array}$ \\
\hline$x^{2}$ & $\begin{array}{l}69,500 \\
73,300 \\
77,200 \\
81,250 \\
85,450\end{array}$ & $\begin{array}{r}92,650 \\
97,700 \\
102,950 \\
108,350 \\
113,950\end{array}$ & $\begin{array}{l}111,200 \\
117,250 \\
123,550 \\
130,050 \\
136,750\end{array}$ & $\begin{array}{l}139,000 \\
146,550 \\
154,400 \\
162,550 \\
170,950\end{array}$ & $\begin{array}{l}166,800 \\
175,850 \\
185,300 \\
195,050 \\
205,150\end{array}$ & $\begin{array}{l}208,450 \\
219,850 \\
231,600 \\
243,800 \\
256,400\end{array}$ & $\begin{array}{l}277,950 \\
293,100 \\
308,800 \\
325,050 \\
341,900\end{array}$ & $\begin{array}{l}416,950 \\
439,700 \\
463,250 \\
487,600 \\
512,850\end{array}$ \\
\hline 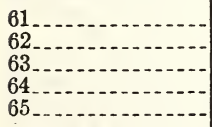 & $\begin{array}{r}89,800 \\
94,300 \\
98,950 \\
103,750 \\
108,650\end{array}$ & $\begin{array}{l}119,750 \\
125,750 \\
131,900 \\
138,300 \\
144,900\end{array}$ & $\begin{array}{l}143,700 \\
150,900 \\
158,300 \\
165,950 \\
173,850\end{array}$ & $\begin{array}{l}179,650 \\
188,600 \\
197,900 \\
207,450 \\
217,350\end{array}$ & $\begin{array}{l}215,550 \\
226,350 \\
237,450 \\
248,950 \\
260,800\end{array}$ & $\begin{array}{l}269,450 \\
282,900 \\
296,850 \\
311,200 \\
326,000\end{array}$ & $\begin{array}{l}359,250 \\
377,250 \\
395,750 \\
414,900 \\
434,700\end{array}$ & $\begin{array}{l}538,900 \\
565,850 \\
593,650 \\
622,400 \\
652,000\end{array}$ \\
\hline $\begin{array}{l}67 \\
68 \\
69 \\
70\end{array}$ & $\begin{array}{l}113,750 \\
119,000 \\
124,400 \\
130,000 \\
135,750\end{array}$ & $\begin{array}{l}151,700 \\
158,700 \\
165,900 \\
173,300 \\
180,950\end{array}$ & $\begin{array}{l}182,000 \\
190,400 \\
199,050 \\
208,000 \\
217,150\end{array}$ & $\begin{array}{l}227,500 \\
238,000 \\
248,850 \\
260,000 \\
271,450\end{array}$ & $\begin{array}{l}273,050 \\
285,650 \\
298,600 \\
312,000 \\
325,750\end{array}$ & $\begin{array}{l}341,300 \\
357,050 \\
373,250 \\
389,950 \\
407,200\end{array}$ & $\begin{array}{l}455,050 \\
476,050 \\
497,700 \\
519,950 \\
542,900\end{array}$ & $\begin{array}{l}682,550 \\
714,050 \\
746,500 \\
779,950 \\
814,350\end{array}$ \\
\hline $\begin{array}{l}71 \\
72 \\
73 \\
74 \\
75\end{array}$ & $\begin{array}{l}141,650 \\
147,700 \\
153,950 \\
160,350 \\
166,950\end{array}$ & $\begin{array}{l}188,850 \\
196,950 \\
205,250 \\
213,800 \\
222,600\end{array}$ & $\begin{array}{l}226,600 \\
236,300 \\
246,300 \\
256,550 \\
267,100\end{array}$ & $\begin{array}{l}283,250 \\
295,400 \\
307,850 \\
320,700 \\
333,850\end{array}$ & $\begin{array}{l}339,900 \\
354,450 \\
369,450 \\
384,850 \\
400,650\end{array}$ & $\begin{array}{l}424,900 \\
443,100 \\
461,800 \\
481,050 \\
500,800\end{array}$ & $\begin{array}{l}566,500 \\
590,800 \\
615,750 \\
641,400 \\
667,750\end{array}$ & $\begin{array}{r}849,750 \\
886,150 \\
923,600 \\
962,100 \\
1,001,600\end{array}$ \\
\hline $\begin{array}{l}76 \\
77 \\
78 \\
79 \\
80\end{array}$ & $\begin{array}{l}173,700 \\
180,650 \\
187,800 \\
195,100 \\
202,600\end{array}$ & $\begin{array}{l}231,600 \\
240,850 \\
250,350 \\
260,150 \\
270,150\end{array}$ & $\begin{array}{l}277,900 \\
289,050 \\
300,450 \\
312,150 \\
324,150\end{array}$ & $\begin{array}{l}347,400 \\
361,300 \\
375,550 \\
390,200 \\
405,200\end{array}$ & $\begin{array}{l}416,900 \\
433,550 \\
450,650 \\
468,250 \\
486,250\end{array}$ & $\begin{array}{l}521,100 \\
541,950 \\
563,350 \\
585,300 \\
607,800\end{array}$ & $\begin{array}{l}694,800 \\
722,600 \\
751,100 \\
780,400 \\
810,400\end{array}$ & $\begin{array}{l}1,042,200 \\
1,083,900 \\
1,126,700 \\
1,170,550 \\
1,215,600\end{array}$ \\
\hline
\end{tabular}


Table 83C.-Resisting moments for poles of woods having an ultimate fiber stress of 5,600 pounds per square inch (western red cedar)

\begin{tabular}{|c|c|c|c|c|c|c|c|c|}
\hline \multirow{2}{*}{$\begin{array}{l}\text { Circumference at } \\
\text { ground line } \\
\text { (inches) }\end{array}$} & \multicolumn{8}{|c|}{ Resisting moments for fiber stress of (pounds per square inch) } \\
\hline & 1,400 & 1,870 & 2,240 & 2,800 & 3,360 & 4,200 & 5,600 & 8,400 \\
\hline 0 & $\begin{array}{r}L b .-f t . \\
2,950\end{array}$ & $\begin{array}{r}L b .-f t . \\
3,950\end{array}$ & $\begin{array}{r}L b .-f t \\
4,750\end{array}$ & $\begin{array}{r}L b .-f t . \\
5,900\end{array}$ & $\begin{array}{r}L b .-f t . \\
7,100\end{array}$ & $\begin{array}{l}L b .-f t . \\
8,850\end{array}$ & $\begin{array}{l}L b .-f t . \\
11,800\end{array}$ & $\begin{array}{l}L b_{.}-\mathrm{ft} . \\
17,750\end{array}$ \\
\hline 5 & $\begin{array}{l}3,400 \\
3,950 \\
4,500 \\
5,100 \\
5,750\end{array}$ & $\begin{array}{l}4,550 \\
5,250 \\
6,000 \\
6,800 \\
7,700\end{array}$ & $\begin{array}{l}5,450 \\
6,300 \\
7,200 \\
8,150 \\
9,250\end{array}$ & $\begin{array}{r}6,850 \\
7,850 \\
9,000 \\
10,200 \\
11,550\end{array}$ & $\begin{array}{r}8,200 \\
9,450 \\
10,800 \\
12,250 \\
13,850\end{array}$ & $\begin{array}{l}10,250 \\
11,800 \\
13,500 \\
15,300 \\
17,300\end{array}$ & $\begin{array}{l}13,700 \\
15,750 \\
17,950 \\
20,400 \\
23,100\end{array}$ & $\begin{array}{l}20,500 \\
23,600 \\
26,950 \\
30,650 \\
34,600\end{array}$ \\
\hline & $\begin{array}{l}6,500 \\
7,250 \\
8,100 \\
9,000 \\
9,950\end{array}$ & $\begin{array}{r}8,650 \\
9,700 \\
10,850 \\
12,050 \\
13,300\end{array}$ & $\begin{array}{l}10,400 \\
11,650 \\
12,950 \\
14,400 \\
15,950\end{array}$ & $\begin{array}{l}13,000 \\
14,550 \\
16,200 \\
18,000 \\
19,950\end{array}$ & $\begin{array}{l}15,600 \\
17,450 \\
19,450 \\
21,600 \\
23,950\end{array}$ & $\begin{array}{l}19,450 \\
21,800 \\
24,300 \\
27,000 \\
29,900\end{array}$ & $\begin{array}{l}25,950 \\
29,100 \\
32,450 \\
36,050 \\
39,900\end{array}$ & $\begin{array}{l}38,950 \\
43,600 \\
48,650 \\
54,050 \\
59,850\end{array}$ \\
\hline & $\begin{array}{l}11,000 \\
12,100 \\
13,250 \\
14,500 \\
15,850\end{array}$ & $\begin{array}{l}14,700 \\
16,150 \\
17,750 \\
19,400 \\
21,150\end{array}$ & $\begin{array}{l}17,600 \\
19,350 \\
21,250 \\
23,250 \\
25,350\end{array}$ & $\begin{array}{l}22,000 \\
24,200 \\
26,550 \\
29,050 \\
31,650\end{array}$ & $\begin{array}{l}26,400 \\
29,050 \\
31,850 \\
34,850 \\
38,000\end{array}$ & $\begin{array}{l}33,000 \\
36,300 \\
39,800 \\
43,550 \\
47,500\end{array}$ & $\begin{array}{l}44,000 \\
48,400 \\
53,100 \\
58,050 \\
63,350\end{array}$ & $\begin{array}{l}66,000 \\
72,600 \\
79,650 \\
87,100 \\
95,000\end{array}$ \\
\hline & $\begin{array}{l}17,250 \\
18,700 \\
20,250 \\
21,900 \\
23,650\end{array}$ & $\begin{array}{l}23,000 \\
25,000 \\
27,050 \\
29,250 \\
31,550\end{array}$ & $\begin{array}{l}27,550 \\
29,950 \\
32,400 \\
35,050 \\
37,800\end{array}$ & $\begin{array}{l}34,450 \\
37,400 \\
40,550 \\
43,800 \\
47,250\end{array}$ & $\begin{array}{l}41,350 \\
44,900 \\
48,650 \\
52,600 \\
56,750\end{array}$ & $\begin{array}{l}51,700 \\
56,100 \\
60,800 \\
65,700 \\
70,900\end{array}$ & $\begin{array}{l}68,900 \\
74,850 \\
81,050 \\
87,650 \\
94,550\end{array}$ & $\begin{array}{l}112,250 \\
121,600 \\
131,450 \\
141,800\end{array}$ \\
\hline 44 & $\begin{array}{l}25,450 \\
27,350 \\
29,350 \\
31,450 \\
33,650\end{array}$ & $\begin{array}{l}34,000 \\
36,550 \\
39,200 \\
42,000 \\
44,950\end{array}$ & & $\begin{array}{l}50,900 \\
54,700 \\
58,750 \\
62,900 \\
67,300\end{array}$ & $\begin{array}{l}61,100 \\
65,650 \\
70,450 \\
75,500 \\
80,750\end{array}$ & $\begin{array}{r}76,350 \\
82,100 \\
88,100 \\
94,400 \\
100,950\end{array}$ & $\begin{array}{l}101,800 \\
109,450 \\
117,450 \\
125,850 \\
134,600\end{array}$ & $\begin{array}{l}152,700 \\
164,150 \\
176,200 \\
188,750 \\
201,950\end{array}$ \\
\hline & $\begin{array}{l}35,950 \\
38,350 \\
40,850 \\
43,450 \\
46,150\end{array}$ & $\begin{array}{l}48,000 \\
51,200 \\
54,550 \\
58,050 \\
61,650\end{array}$ & $\begin{array}{l}57,500 \\
61,350 \\
65,350 \\
69,500 \\
73,850\end{array}$ & $\begin{array}{l}71,900 \\
76,700 \\
81,700 \\
86,900 \\
92,350\end{array}$ & $\begin{array}{r}86,300 \\
92,050 \\
98,050 \\
104,300 \\
110,800\end{array}$ & $\begin{array}{l}107,850 \\
115,050 \\
122,550 \\
130,350 \\
138,500\end{array}$ & $\begin{array}{l}143,800 \\
153,400 \\
163,400 \\
173,800 \\
184,650\end{array}$ & $\begin{array}{l}215,700 \\
230,050 \\
245,050 \\
260,700 \\
277,000\end{array}$ \\
\hline $\begin{array}{l}51 . \\
52 . \\
53 \\
54\end{array}$ & $\begin{array}{l}49,000 \\
51,950 \\
55,000 \\
58,150 \\
61,450\end{array}$ & $\begin{array}{l}65,450 \\
69,350 \\
73,450 \\
77,700 \\
82,050\end{array}$ & $\begin{array}{l}78,400 \\
83,100 \\
87,950 \\
93,050 \\
98,300\end{array}$ & $\begin{array}{r}98,000 \\
103,850 \\
109,950 \\
116,300 \\
122,900\end{array}$ & & & & $\begin{array}{l}293,950 \\
311,600 \\
329,900 \\
348,950 \\
368,650\end{array}$ \\
\hline $\begin{array}{l}56 . \\
57 . \\
58 . \\
59 .\end{array}$ & $\begin{array}{l}64,850 \\
68,400 \\
72,050 \\
75,850 \\
79,750\end{array}$ & $\begin{array}{r}86,650 \\
91,350 \\
96,250 \\
101,300 \\
106,550\end{array}$ & $\begin{array}{l}103,750 \\
109,450 \\
115,300 \\
121,350 \\
127,650\end{array}$ & $\begin{array}{l}129,700 \\
136,800 \\
144,100 \\
151,700 \\
159,550\end{array}$ & $\begin{array}{l}155,650 \\
164,150 \\
172,950 \\
182,050 \\
191,450\end{array}$ & $\begin{array}{l}194,600 \\
205,200 \\
216,200 \\
227,550 \\
239,300\end{array}$ & $\begin{array}{l}259,450 \\
273,600 \\
288,250 \\
303,400 \\
319,100\end{array}$ & $\begin{array}{l}389,150 \\
410,350 \\
432,350 \\
455,100 \\
478,650\end{array}$ \\
\hline $\begin{array}{l}61 . \\
62- \\
63 . \\
64- \\
65 .\end{array}$ & $\begin{array}{r}83,850 \\
88,000 \\
92,350 \\
96,800 \\
101,400\end{array}$ & $\begin{array}{l}111,950 \\
117,550 \\
123,350 \\
129,300 \\
135,450\end{array}$ & $\begin{array}{l}134,150 \\
140,850 \\
147,750 \\
154,900 \\
162,300\end{array}$ & $\begin{array}{l}167,650 \\
176,050 \\
184,700 \\
193,600 \\
202,850\end{array}$ & $\begin{array}{l}201,200 \\
211,250 \\
221,650 \\
232,350 \\
243,400\end{array}$ & $\begin{array}{l}251,500 \\
264,050 \\
277,050 \\
290,450 \\
304,250\end{array}$ & $\begin{array}{l}335,300 \\
352,100 \\
369,400 \\
387,250 \\
405,700\end{array}$ & $\begin{array}{l}502,950 \\
528,100 \\
554,100 \\
580,900 \\
608,550\end{array}$ \\
\hline
\end{tabular}


Table 83C.-Resisting moments for poles of woods having an ultimate fiber stress of 5,600 pounds per square inch (western red cedar)-Contd.

\begin{tabular}{|c|c|c|c|c|c|c|c|c|}
\hline \multirow{2}{*}{$\begin{array}{l}\text { Circumference at } \\
\text { ground line } \\
\text { (inches) }\end{array}$} & \multicolumn{8}{|c|}{ Resisting moments for fiber stress of (pounds per square inch) } \\
\hline & 1,400 & 1,870 & 2,240 & 2,800 & 3,360 & 4,200 & 5,600 & 8,400 \\
\hline 69 & $\begin{array}{c}\text { Lb.-ft. } \\
106,200 \\
111,100 \\
116,150 \\
121,300 \\
126,700\end{array}$ & $\begin{array}{c}\text { Lb.-ft. } \\
141,800 \\
148,350 \\
155,100 \\
162,050 \\
169,200\end{array}$ & $\begin{array}{c}\text { Lb.-ft. } \\
169,900 \\
177,700 \\
185,800 \\
194,100 \\
202,700\end{array}$ & $\begin{array}{c}L b .-f t . \\
212,350 \\
222,150 \\
232,250 \\
242,650 \\
253,350\end{array}$ & $\begin{array}{c}L b .-f t . \\
254,850 \\
266,600 \\
278,700 \\
291,200 \\
304,000\end{array}$ & $\begin{array}{c}\text { Lb.-ft. } \\
318,550 \\
333,250 \\
348,400 \\
363,950 \\
380,050\end{array}$ & $\begin{array}{c}\text { Lb.-ft. } \\
424,700 \\
444,300 \\
464,500 \\
485,300 \\
506,700\end{array}$ & $\begin{array}{l}L b .-f t . \\
637,050 \\
666,450 \\
696,750 \\
727,950 \\
760,050\end{array}$ \\
\hline $\begin{array}{l}73 \\
74 \\
75\end{array}$ & $\begin{array}{l}132,200 \\
137,850 \\
143,650 \\
149,650 \\
155,800\end{array}$ & $\begin{array}{l}176,550 \\
184,150 \\
191,900 \\
199,900 \\
208,100\end{array}$ & $\begin{array}{l}211,500 \\
220,550 \\
229,850 \\
239,450 \\
249,300\end{array}$ & $\begin{array}{l}264,350 \\
275,700 \\
287,350 \\
299,300 \\
311,600\end{array}$ & $\begin{array}{l}317,250 \\
330,850 \\
344,800 \\
359,200 \\
373,950\end{array}$ & $\begin{array}{l}396,550 \\
413,550 \\
431,000 \\
448,950 \\
467,400\end{array}$ & $\begin{array}{l}528,750 \\
551,400 \\
574,700 \\
598,650 \\
623,250\end{array}$ & $\begin{array}{l}793,100 \\
827,100 \\
862,050 \\
897,950 \\
934,850\end{array}$ \\
\hline $\begin{array}{l}76 \\
77 \\
79 \\
80\end{array}$ & $\begin{array}{l}162,100 \\
168,600 \\
175,250 \\
182,100 \\
189,100\end{array}$ & $\begin{array}{l}216,550 \\
225,200 \\
234,100 \\
243,200 \\
252,550\end{array}$ & $\begin{array}{l}259,400 \\
269,750 \\
280,400 \\
291,350 \\
302,550\end{array}$ & $\begin{array}{l}324,250 \\
337,200 \\
350,500 \\
364,200 \\
378,200\end{array}$ & $\begin{array}{l}389,100 \\
404,650 \\
420,650 \\
437,000 \\
453,800\end{array}$ & $\begin{array}{l}486,350 \\
505,800 \\
525,800 \\
546,250 \\
567,300\end{array}$ & $\begin{array}{l}648,500 \\
674,450 \\
701,050 \\
728,350 \\
756,350\end{array}$ & $\begin{array}{r}972,750 \\
1,011,650 \\
1,051,550 \\
1,092,550 \\
1,134,550\end{array}$ \\
\hline
\end{tabular}

Table 84.- Resisting moments for poles of woods with an ultimate fiber stress of 5,000 pounds per square inch (other yellow pine, cypress)

\begin{tabular}{|c|c|c|c|c|c|c|c|c|}
\hline \multirow{2}{*}{$\begin{array}{l}\text { Circumference at } \\
\text { ground line } \\
\text { (inches) }\end{array}$} & \multicolumn{8}{|c|}{ Resisting moments for fiber stress of (pounds per square inch) } \\
\hline & 1,250 & 1,670 & 2,000 & 2,500 & 3,000 & 3,750 & 5,000 & 7,500 \\
\hline 20. & $\begin{array}{r}L b .-f t . \\
2,650\end{array}$ & $\begin{array}{r}L b .-f t . \\
3,500\end{array}$ & $\begin{array}{l}L b .-f t . \\
4,200\end{array}$ & $\begin{array}{r}L b .-f t . \\
5,300\end{array}$ & $\begin{array}{r}L b .-f t . \\
6,350\end{array}$ & $\begin{array}{l}L b .-f t . \\
7,900\end{array}$ & $\begin{array}{l}L b .-f t . \\
10,550\end{array}$ & $\begin{array}{l}L b .-f t . \\
15,850\end{array}$ \\
\hline $\begin{array}{l}21 . \\
22 . \\
23 . \\
24 . \\
25 .\end{array}$ & $\begin{array}{l}3,050 \\
3,500 \\
4,000 \\
4,550 \\
5,150\end{array}$ & $\begin{array}{l}4,100 \\
4,700 \\
5,350 \\
6,100 \\
6,900\end{array}$ & $\begin{array}{l}4,900 \\
5,600 \\
6,400 \\
7,300 \\
8,250\end{array}$ & $\begin{array}{r}6,100 \\
7,000 \\
8,000 \\
9,100 \\
10,300\end{array}$ & $\begin{array}{r}7,350 \\
8,450 \\
9,650 \\
10,950 \\
12,350\end{array}$ & $\begin{array}{r}9,150 \\
10,550 \\
12,050 \\
13,700 \\
15,450\end{array}$ & $\begin{array}{l}12,200 \\
14,050 \\
16,050 \\
18,250 \\
20,600\end{array}$ & $\begin{array}{l}18,300 \\
21,050 \\
24,050 \\
27,350 \\
30,900\end{array}$ \\
\hline $\begin{array}{l}28 . \\
30 . \\
30 .\end{array}$ & $\begin{array}{l}5,800 \\
6,500 \\
7,250 \\
8,050 \\
8,900\end{array}$ & $\begin{array}{r}7,750 \\
8,650 \\
9,650 \\
10,750 \\
11,960\end{array}$ & $\begin{array}{r}9,250 \\
10,400 \\
11,600 \\
12,850 \\
14,250\end{array}$ & $\begin{array}{l}11,600 \\
13,000 \\
14,500 \\
16,100 \\
17,800\end{array}$ & $\begin{array}{l}13,900 \\
15,600 \\
17,350 \\
19,300 \\
21,350\end{array}$ & $\begin{array}{l}17,400 \\
19,450 \\
21,700 \\
24,150 \\
26,700\end{array}$ & $\begin{array}{l}23,200 \\
25,950 \\
28,950 \\
32,150 \\
35,600\end{array}$ & $\begin{array}{l}34,750 \\
38,950 \\
43,450 \\
48,250 \\
53,400\end{array}$ \\
\hline $\begin{array}{l}31 . \\
32- \\
33- \\
34-\end{array}$ & $\begin{array}{r}9,800 \\
10,800 \\
11,850 \\
12,950 \\
14,150\end{array}$ & $\begin{array}{l}13,100 \\
14,450 \\
15,850 \\
17,300 \\
18,900\end{array}$ & $\begin{array}{l}15,700 \\
17,300 \\
18,950 \\
20,750 \\
22,600\end{array}$ & $\begin{array}{l}19,650 \\
21,600 \\
23,700 \\
25,900 \\
28,300\end{array}$ & $\begin{array}{l}23,600 \\
25,950 \\
28,450 \\
31,100 \\
33,950\end{array}$ & $\begin{array}{l}29,450 \\
32,400 \\
35,550 \\
38,900 \\
42,400\end{array}$ & $\begin{array}{l}39,300 \\
43,200 \\
47,400 \\
51,850 \\
56,550\end{array}$ & $\begin{array}{l}58,950 \\
64,850 \\
71,100 \\
77,750 \\
84,850\end{array}$ \\
\hline
\end{tabular}


Table 84.- Resisting moments for poles of woods with an ultimate fiber stress of 5,000 pounds per square inch (other yellow pine, cypress)Continued

\begin{tabular}{|c|c|c|c|c|c|c|c|c|}
\hline \multirow{2}{*}{$\begin{array}{l}\text { Circumference at } \\
\text { ground line } \\
\text { (inches) }\end{array}$} & \multicolumn{8}{|c|}{ Resisting moments for fiber stress of (pounds per square inch) } \\
\hline & 1,250 & 1,670 & 2,000 & 2,500 & 3,000 & 3,750 & 5,000 & 7,500 \\
\hline $\begin{array}{l}36 \\
37 \\
38\end{array}$ & $\begin{array}{l}\text { Lb. ft. } \\
15,400 \\
16,700 \\
18,100 \\
19,550 \\
21,100\end{array}$ & $\begin{array}{l}L b .-f t . \\
20,550 \\
22,300 \\
24,150 \\
26,150 \\
28,200\end{array}$ & $\begin{array}{l}L b .-f t . \\
24,600 \\
26,700 \\
28,950 \\
31,300 \\
33,750\end{array}$ & $\begin{array}{l}\text { Lb. fft. } \\
30,750 \\
33,400 \\
36,200 \\
39,100 \\
42,200\end{array}$ & $\begin{array}{l}\text { Lb. } f t . \\
36,900 \\
40,100 \\
43,450 \\
46,950 \\
50,650\end{array}$ & $\begin{array}{l}L b .-f t . \\
46,150 \\
50,100 \\
54,300 \\
58,700 \\
63,300\end{array}$ & $\begin{array}{l}\text { Lb.-ft. } \\
61,550 \\
66,800 \\
72,400 \\
78,250 \\
84,400\end{array}$ & $\begin{array}{r}L b .-f t . \\
92,300 \\
100,200 \\
108,550 \\
117,350 \\
126,600\end{array}$ \\
\hline 41 & $\begin{array}{l}22,750 \\
24,450 \\
26,200 \\
28,100 \\
30,050\end{array}$ & $\begin{array}{l}30,350 \\
32,650 \\
35,050 \\
37,550 \\
40,150\end{array}$ & $\begin{array}{l}36,350 \\
39,100 \\
41,950 \\
44,950 \\
48,100\end{array}$ & $\begin{array}{l}45,450 \\
48,850 \\
52,450 \\
56,200 \\
60,100\end{array}$ & $\begin{array}{l}54,550 \\
58,650 \\
62,900 \\
67,400 \\
72,100\end{array}$ & $\begin{array}{l}68,200 \\
73,300 \\
78,650 \\
84,250 \\
90,150\end{array}$ & $\begin{array}{r}90,900 \\
97,700 \\
104,850 \\
112,3 \approx 0 \\
120,200\end{array}$ & $\begin{array}{l}136,350 \\
146,600 \\
157,300 \\
168,550 \\
180,300\end{array}$ \\
\hline 16 & $\begin{array}{l}32,100 \\
34,250 \\
36,450 \\
38,800 \\
41,200\end{array}$ & $\begin{array}{l}42,900 \\
45,750 \\
48,700 \\
51,850 \\
55,050\end{array}$ & $\begin{array}{l}51,350 \\
54,800 \\
58,350 \\
62,050 \\
65,950\end{array}$ & $\begin{array}{l}64,200 \\
68,450 \\
72,950 \\
77,600 \\
82,450\end{array}$ & $\begin{array}{l}77,050 \\
82,150 \\
87,500 \\
93,100 \\
98,950\end{array}$ & $\begin{array}{r}96,300 \\
102,700 \\
109,400 \\
116,400 \\
123,650\end{array}$ & $\begin{array}{l}128,400 \\
136,950 \\
145,850 \\
155,200 \\
164,900\end{array}$ & $\begin{array}{l}192,600 \\
205,400 \\
218,800 \\
232,750 \\
247,300\end{array}$ \\
\hline & $\begin{array}{l}43,750 \\
46,350 \\
49,100 \\
51,900 \\
54,850\end{array}$ & $\begin{array}{l}58,450 \\
61,950 \\
65,600 \\
69,350 \\
73,300\end{array}$ & $\begin{array}{l}70,000 \\
74,200 \\
78,550 \\
83,100 \\
87,800\end{array}$ & $\begin{array}{r}87,500 \\
92,750 \\
98,200 \\
103,850 \\
109,700\end{array}$ & $\begin{array}{l}105,000 \\
111,300 \\
117,800 \\
124,600 \\
131,650\end{array}$ & $\begin{array}{l}131,250 \\
139,100 \\
147,300 \\
155,750 \\
164,600\end{array}$ & $\begin{array}{l}174,950 \\
185,450 \\
196,350 \\
207,700 \\
219,450\end{array}$ & $\begin{array}{l}262,450 \\
278,200 \\
294,550 \\
311,550 \\
329,150\end{array}$ \\
\hline & $\begin{array}{l}57,900 \\
61,050 \\
64,350 \\
67,700 \\
71,250\end{array}$ & $\begin{array}{l}77,350 \\
81,600 \\
85,950 \\
90,500 \\
95,150\end{array}$ & $\begin{array}{r}92,650 \\
97,700 \\
102,950 \\
108,350 \\
113,950\end{array}$ & $\begin{array}{l}115,800 \\
122,150 \\
128,700 \\
135,450 \\
142,450\end{array}$ & $\begin{array}{l}139,000 \\
146,550 \\
154,400 \\
162,550 \\
170,950\end{array}$ & $\begin{array}{l}173,750 \\
183,200 \\
193,000 \\
203,150 \\
213,700\end{array}$ & $\begin{array}{l}231,650 \\
244,250 \\
257,350 \\
270,900 \\
284,900\end{array}$ & $\begin{array}{l}347,450 \\
366,400 \\
386,050 \\
406,350 \\
427,350\end{array}$ \\
\hline & $\begin{array}{l}74,850 \\
78,600 \\
82,450 \\
86,450 \\
90,550\end{array}$ & $\begin{array}{l}100,000 \\
105,000 \\
110,150 \\
115,500 \\
121,000\end{array}$ & $\begin{array}{l}119,750 \\
125,750 \\
131,900 \\
138,300 \\
144,900\end{array}$ & $\begin{array}{l}149,700 \\
157,200 \\
164,900 \\
172,900 \\
181,100\end{array}$ & $\begin{array}{l}179,650 \\
188,600 \\
197,900 \\
207,450 \\
217,350\end{array}$ & $\begin{array}{l}224,550 \\
235,750 \\
247,350 \\
259,350 \\
271,650\end{array}$ & $\begin{array}{l}299,400 \\
314,350 \\
329,800 \\
345,750 \\
362,250\end{array}$ & $\begin{array}{l}449,100 \\
471,550 \\
494,700 \\
518,650 \\
543,350\end{array}$ \\
\hline & $\begin{array}{r}94,800 \\
99,200 \\
103,700 \\
108,350 \\
113,100\end{array}$ & $\begin{array}{l}126,650 \\
132,500 \\
138,500 \\
144,700 \\
151,100\end{array}$ & $\begin{array}{l}151,700 \\
158,700 \\
165,900 \\
173,300 \\
180,950\end{array}$ & $\begin{array}{l}189,600 \\
198,350 \\
207,350 \\
216,650 \\
226,200\end{array}$ & $\begin{array}{l}227,500 \\
238,000 \\
248,850 \\
260,000 \\
271,450\end{array}$ & $\begin{array}{l}284,400 \\
297,550 \\
311,050 \\
325,000 \\
339,300\end{array}$ & $\begin{array}{l}379,200 \\
396,700 \\
414,750 \\
433,300 \\
452,400\end{array}$ & $\begin{array}{l}568,800 \\
595,050 \\
622,100 \\
649,950 \\
678,650\end{array}$ \\
\hline & $\begin{array}{l}118,000 \\
123,100 \\
128,300 \\
133,600 \\
139,100\end{array}$ & $\begin{array}{l}157,700 \\
164,450 \\
171,400 \\
178,500 \\
185,850\end{array}$ & $\begin{array}{l}188,850 \\
196,950 \\
205,250 \\
213,800 \\
222,600\end{array}$ & $\begin{array}{l}236,050 \\
246,150 \\
256,550 \\
267,250 \\
278,250\end{array}$ & $\begin{array}{l}283,250 \\
295,400 \\
307,850 \\
320,700 \\
333,850\end{array}$ & $\begin{array}{l}354,050 \\
369,250 \\
384,850 \\
400,850 \\
417,350\end{array}$ & $\begin{array}{l}472,100 \\
492,300 \\
513,100 \\
534,500 \\
556,450\end{array}$ & $\begin{array}{l}708,150 \\
738,450 \\
769,650 \\
801,750 \\
834,700\end{array}$ \\
\hline $\begin{array}{l}77 . \\
78 \\
79 \\
80\end{array}$ & $\begin{array}{l}144,750 \\
150,550 \\
156,500 \\
162,600 \\
168,850\end{array}$ & $\begin{array}{l}193,400 \\
201,100 \\
209,050 \\
217,200 \\
225,550\end{array}$ & $\begin{array}{l}231,600 \\
240,850 \\
250,350 \\
260,150 \\
270,150\end{array}$ & $\begin{array}{l}289,500 \\
301,100 \\
312,950 \\
325,150 \\
337,650\end{array}$ & $\begin{array}{l}347,400 \\
361,300 \\
375,550 \\
390,200 \\
405,200\end{array}$ & $\begin{array}{l}434,250 \\
451,650 \\
469,450 \\
487,750 \\
506,500\end{array}$ & $\begin{array}{l}579,000 \\
602,150 \\
625,950 \\
650,300 \\
675,350\end{array}$ & $\begin{array}{r}868,500 \\
903,250 \\
938,900 \\
975,500 \\
1,013,000\end{array}$ \\
\hline
\end{tabular}


Table 85.-Resisting moments for poles of woods having ultimate fiber stress of 3,600 pounds per square inch (northern white cedar, redwood)

\begin{tabular}{|c|c|c|c|c|c|c|c|c|}
\hline \multirow{2}{*}{$\begin{array}{l}\text { Circumference at } \\
\text { ground line } \\
\text { (inches) }\end{array}$} & \multicolumn{8}{|c|}{ Resisting moments for fiber stress of (pounds per square inch)- } \\
\hline & 900 & 1,200 & 1,440 & 1,800 & 2,160 & 2,700 & 3,600 & 5,400 \\
\hline - & $\begin{array}{r}L b .-f t \\
1,900\end{array}$ & $\begin{array}{r}L b .-f t . \\
2,550\end{array}$ & $\begin{array}{l}L b .-f t . \\
3,050\end{array}$ & $\begin{array}{l}\text { Lb.-ft. } \\
3,800\end{array}$ & $\begin{array}{r}L b .-f t . \\
4,550\end{array}$ & $\begin{array}{r}L b .-f t . \\
5,700\end{array}$ & $\begin{array}{r}L b .-f t . \\
7,600\end{array}$ & $\begin{array}{l}L b .-f t . \\
11,400\end{array}$ \\
\hline 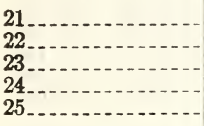 & $\begin{array}{l}2,200 \\
2,550 \\
2,900 \\
3,300 \\
3,700\end{array}$ & $\begin{array}{l}2,950 \\
3,350 \\
3,850 \\
4,400 \\
4,950\end{array}$ & $\begin{array}{l}3,500 \\
4,050 \\
4,600 \\
5,250 \\
5,950\end{array}$ & $\begin{array}{l}4,400 \\
5,050 \\
5,800 \\
6,550 \\
7,400\end{array}$ & $\begin{array}{l}5,300 \\
6,050 \\
6,950 \\
7,900 \\
8,900\end{array}$ & $\begin{array}{r}6,600 \\
7,600 \\
8,650 \\
9,850 \\
11,150\end{array}$ & $\begin{array}{r}8,800 \\
10,100 \\
11,550 \\
13,150 \\
14,850\end{array}$ & $\begin{array}{l}13,200 \\
15,150 \\
17,350 \\
19,700 \\
22,250\end{array}$ \\
\hline 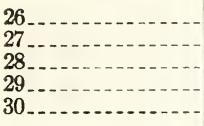 & $\begin{array}{l}4,150 \\
4,650 \\
5,200 \\
5,800 \\
6,400\end{array}$ & $\begin{array}{l}5,550 \\
6,250 \\
6,950 \\
7,700 \\
8,550\end{array}$ & $\begin{array}{r}6,700 \\
7,500 \\
8,350 \\
9,250 \\
10,250\end{array}$ & $\begin{array}{r}8,350 \\
9,350 \\
10,400 \\
11,600 \\
12,800\end{array}$ & $\begin{array}{l}10,000 \\
11,200 \\
12,500 \\
13,900 \\
15,400\end{array}$ & $\begin{array}{l}12,500 \\
14,000 \\
15,650 \\
17,350 \\
19,250\end{array}$ & $\begin{array}{l}16,700 \\
18,700 \\
20,850 \\
23,150 \\
25,650\end{array}$ & $\begin{array}{l}25,050 \\
28,050 \\
31,250 \\
34,750 \\
38,450\end{array}$ \\
\hline 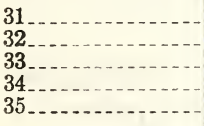 & $\begin{array}{r}7,050 \\
7,800 \\
8,550 \\
9,350 \\
10,200\end{array}$ & $\begin{array}{r}9,450 \\
10,350 \\
11,400 \\
12,450 \\
13,550\end{array}$ & & & & $\begin{array}{l}21,200 \\
23,350 \\
25,600 \\
28,000 \\
30,550\end{array}$ & & $\begin{array}{l}42,450 \\
46,700 \\
51,200 \\
56,000 \\
61,100\end{array}$ \\
\hline $40_{--}$ & $\begin{array}{l}11,100 \\
12,050 \\
13,050 \\
14,105 \\
15,200\end{array}$ & $\begin{array}{l}14,750 \\
16,050 \\
17,350 \\
18,800 \\
20,250\end{array}$ & & & & & & $\begin{array}{l}66,450 \\
72,150 \\
78,150 \\
84,500 \\
91,150\end{array}$ \\
\hline IU - & $\begin{array}{l}16,350 \\
17,600 \\
18,900 \\
20,200 \\
21,650\end{array}$ & $\begin{array}{l}21,800 \\
23,450 \\
25,150 \\
26,950 \\
28,850\end{array}$ & & & & & & $\begin{array}{r}98,200 \\
105,550 \\
113,250 \\
121,350 \\
129,800\end{array}$ \\
\hline 50 & $\begin{array}{l}23,100 \\
24,650 \\
26,250 \\
27,950 \\
29,700\end{array}$ & $\begin{array}{l}30,800 \\
32,850 \\
35,000 \\
37,250 \\
39,550\end{array}$ & & & & & & $\begin{array}{l}138,650 \\
147,900 \\
157,550 \\
167,600 \\
178,050\end{array}$ \\
\hline $55 \ldots$ & $\begin{array}{l}31,500 \\
33,400 \\
35,350 \\
37,400 \\
39,500\end{array}$ & & & $\begin{array}{c}63,000 \\
66,750 \\
70,700 \\
74,750 \\
79,000\end{array}$ & $\begin{array}{l}75,600 \\
80,100 \\
84,850 \\
89,700 \\
94,800\end{array}$ & $\begin{array}{r}94,500 \\
100,150 \\
106,050 \\
112,150 \\
118,500\end{array}$ & $\begin{array}{l}126,000 \\
133,550 \\
141,400 \\
149,550 \\
158,000\end{array}$ & $\begin{array}{l}188,950 \\
200,300 \\
212,100 \\
224,300 \\
237,000\end{array}$ \\
\hline $00 \ldots$ & $\begin{array}{l}41,700 \\
43,950 \\
46,300 \\
48,750 \\
51,300\end{array}$ & $\begin{array}{l}55,600 \\
58,600 \\
61,750 \\
65,000 \\
68,400\end{array}$ & $\begin{array}{l}66,700 \\
70,350 \\
74,100 \\
78,000 \\
82,050\end{array}$ & $\begin{array}{r}83,400 \\
87,950 \\
92,650 \\
97,500 \\
102,550\end{array}$ & $\begin{array}{l}100,050 \\
105,500 \\
111,200 \\
117,050 \\
123,100\end{array}$ & $\begin{array}{l}125,100 \\
131,900 \\
138,950 \\
146,300 \\
153,850\end{array}$ & $\begin{array}{l}166,800 \\
175,850 \\
185,300 \\
195,050 \\
205,150\end{array}$ & $\begin{array}{l}250,150 \\
263,800 \\
277,950 \\
292,550 \\
307,700\end{array}$ \\
\hline & $\begin{array}{l}53,900 \\
56,800 \\
59,350 \\
62,250 \\
65,200\end{array}$ & $\begin{array}{l}71,850 \\
75,450 \\
79,150 \\
83,000 \\
86,950\end{array}$ & $\begin{array}{r}86,200 \\
90,550 \\
95,000 \\
99,605 \\
104,300\end{array}$ & $\begin{array}{l}107,800 \\
113,150 \\
118,750 \\
124,500 \\
130,400\end{array}$ & $\begin{array}{l}129,350 \\
135,800 \\
142,500 \\
149,350 \\
156,500\end{array}$ & $\begin{array}{l}161,650 \\
169,750 \\
178,100 \\
186,700 \\
195,600\end{array}$ & $\begin{array}{l}215,550 \\
226,350 \\
237,450 \\
248,950 \\
260,800\end{array}$ & $\begin{array}{l}323,350 \\
339,500 \\
356,200 \\
373,450 \\
391,200\end{array}$ \\
\hline 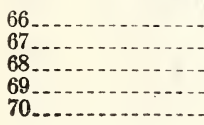 & $\begin{array}{l}68,250 \\
71,400 \\
74,650 \\
78,000 \\
81,450\end{array}$ & $\begin{array}{r}91,000 \\
95,200 \\
99,550 \\
104,000 \\
108,600\end{array}$ & $\begin{array}{l}109,200 \\
114,250 \\
119,450 \\
124,800 \\
130,300\end{array}$ & $\begin{array}{l}136,500 \\
142,800 \\
149,300 \\
156,000 \\
162,850\end{array}$ & $\begin{array}{l}163,800 \\
171,400 \\
179,150 \\
187,200 \\
195,450\end{array}$ & $\begin{array}{l}204,750 \\
214,200 \\
223,950 \\
234,000 \\
244,300\end{array}$ & $\begin{array}{l}273,050 \\
285,650 \\
298,600 \\
312,000 \\
325,750\end{array}$ & $\begin{array}{l}409,550 \\
428,450 \\
447,900 \\
467,950 \\
488,600\end{array}$ \\
\hline
\end{tabular}


Table 85.-Resisting moments for poles of woods having ultimate fiber stress of 3,600 pounds per square inch (northern white cedar, redwood)Continued

\begin{tabular}{|c|c|c|c|c|c|c|c|c|}
\hline \multirow{2}{*}{$\begin{array}{l}\text { Circumference at } \\
\text { ground line } \\
\text { (inches) }\end{array}$} & \multicolumn{8}{|c|}{ Resisting moments for fiber stress of (pounds per square inch)- } \\
\hline & 900 & 1,200 & 1,440 & 1,800 & 2,160 & 2,700 & 3,600 & 5,400 \\
\hline & $\begin{array}{r}L b .-f t . \\
85,000 \\
88,600 \\
92,350 \\
96,200 \\
100,150\end{array}$ & $\begin{array}{c}L b .-f t . \\
113,300 \\
118,150 \\
123,150 \\
128,300 \\
133,550\end{array}$ & $\begin{array}{c}L b .-f t . \\
135,950 \\
141,800 \\
147,800 \\
153,950 \\
160,250\end{array}$ & $\begin{array}{c}L b .-f t . \\
169,950 \\
177,250 \\
184,700 \\
192,400 \\
200,300\end{array}$ & $\begin{array}{c}L b .-f t . \\
203,950 \\
212,700 \\
221,650 \\
230,900 \\
240,400\end{array}$ & $\begin{array}{c}L b .-f t . \\
254,950 \\
265,850 \\
277,100 \\
288,600 \\
300,500\end{array}$ & $\begin{array}{c}\text { Lb.-ft. } \\
339,900 \\
354,450 \\
369,450 \\
384,850 \\
400,650\end{array}$ & $\begin{array}{l}L b .-f t . \\
509,850 \\
531,700 \\
554,150 \\
577,250 \\
600,950\end{array}$ \\
\hline 76 & $\begin{array}{l}104,200 \\
108,400 \\
112,650 \\
117,050 \\
121,550\end{array}$ & $\begin{array}{l}138,950 \\
144,500 \\
150,200 \\
156,100 \\
162,100\end{array}$ & $\begin{array}{l}166,750 \\
173,400 \\
180,250 \\
187,300 \\
194,500\end{array}$ & $\begin{array}{l}208,450 \\
216,800 \\
225,350 \\
234,100 \\
243,100\end{array}$ & $\begin{array}{l}250,150 \\
260,150 \\
270,400 \\
280,950 \\
291,750\end{array}$ & $\begin{array}{l}312,650 \\
325,150 \\
338,000 \\
351,150 \\
364,700\end{array}$ & $\begin{array}{l}416,900 \\
433,550 \\
450,650 \\
468,250 \\
486,250\end{array}$ & $\begin{array}{l}625,350 \\
650,350 \\
676,000 \\
702,350 \\
729,350\end{array}$ \\
\hline
\end{tabular}

When pole timbers are used that have ultimate strengths different from those listed, the dimensions of the poles of grades $\mathrm{A}, \mathrm{B}$, or $\mathrm{C}$ to withstand the vertical and transverse loads assumed in the National Electrical Safety Code, section 25 , may be determined by application of the ratios of ultimate fiber stress of the wood to allowable fiber stress as installed under the various conditions stipulated in Table 87A.

Table 87A. - Ratio of ultimate fiber stresses of wood poles to allowable fiber stresses under vertical and transverse loading, grade A, B, or C

\begin{tabular}{|c|c|c|c|}
\hline \multirow[b]{2}{*}{ Situation and grade } & \multicolumn{2}{|c|}{ When installed } & \multirow{2}{*}{ 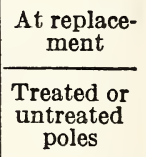 } \\
\hline & $\begin{array}{c}\text { Treated } \\
\text { poles }\end{array}$ & $\begin{array}{c}\text { Untreated } \\
\text { poles }\end{array}$ & \\
\hline $\begin{array}{l}\text { At crossings: } \\
\text { Poles in lines of one grade of construction through- } \\
\text { out- } \\
\text { Grade A } \\
\text { Grade B B } \\
\text { Grade C } \\
\text { Poles in isolated sections of higher grade of con- } \\
\text { struction in lines of a lower grade of construc- } \\
\text { tion- } \\
\text { Grade A } \\
\text { Grade B } \\
\text { Grade C } \\
\text { Elsewhere than at crossings: } \\
\text { Grade A } \\
\text { Grade B } \\
\text { Grade C }\end{array}$ & \begin{tabular}{l|}
3 \\
2 \\
$11 / 3$ \\
$21 / 2$ \\
$12 / 3$ \\
1
\end{tabular} & $\begin{array}{l}4 \\
3 \\
123 \\
3 \\
2 \\
113\end{array}$ & $\begin{array}{l}13 / 3 \\
1_{33}\end{array}$ \\
\hline
\end{tabular}




\section{TRANSVERSE LOADS ON POLES}

Transverse and vertical loads on poles of grades A, B, C, $\mathrm{D}$, or $\mathrm{E}$ are assumed to act simultaneously. The fiber stresses caused by the transverse wind forces on poles and wires must therefore be added to the fiber stresses caused by the weights of the loaded conductors supported when determining the safety of the pole.

Transverse loads on a line result in a bending moment on unguyed poles. The bending moment at the ground line is determined by multiplying the transverse force resulting from the assumed loads of section 25, by the height of the transverse force.

The transverse force acting on the conductors in lines of grades $\mathrm{A}, \mathrm{B}, \mathrm{C}, \mathrm{D}$, and $\mathrm{E}$ for any given loading district may be found by use of the values of transverse loads on conductors per foot of length given in National Electrical Safety Code, Table 82 . The transverse force in pounds contributed by each conductor is obtained by multiplying the transverse force per foot by the span length in feet or by one-half the sum of the adjacent spans. The moment in pound-feet due to the transverse force on each conductor, acting at the ground line of the pole, is found by multiplying this force by the height in feet at which it is applied. The total moment in pound-feet acting at the ground line of the pole is the sum of the moments resulting from conductors or messengers contributing to the transverse load under the loading assumptions, and the moment resulting from the transverse wind pressure on the pole itself. The moment in pound-feet resulting from the transverse wind force on the pole itself, assuming a pole of circular cross section tapering uniformly, may be obtained from Table 87B, or from the following formulas:

For heavy and medium loading, $M=4(D+2 d) h^{2} / 3$ poundfeet.

For light loading, $M=2(D+2 d) h^{2}$ pound-feet. where

$D=$ diameter at ground line in feet.

$d=$ diameter at top in feet.

$h=$ height of pole in feet. 
Table 87B.-Moments due to wind pressure on poles

HEAVY AND MEDIUM LOADING

\begin{tabular}{c|r|r|r|r|r|r|r}
\hline $\begin{array}{l}\text { T= Top circum- } \\
\text { ference in } \\
\text { inches }\end{array}$ \\
$\begin{array}{c}\text { G= Ground-line } \\
\text { circumference } \\
\text { in inches }\end{array}$
\end{tabular}


WOOD POLES FOR OVERHEAD ELECTRICAL LINES 17

Table 87B.-Moments due to wind pressure on poles-Continued HEAVY AND MEDIUM LOADING-Continued

\begin{tabular}{|c|c|c|c|c|c|c|}
\hline \multirow{2}{*}{$\begin{array}{l}T=\text { Top circumference in } \\
\text { inches } \\
G=\text { Ground-line circumfer- } \\
\text { ence in inches }\end{array}$} & \multicolumn{6}{|c|}{$\begin{array}{l}\text { Moment due to wind pressure on poles of various heights } \\
\text { above ground }\end{array}$} \\
\hline & 48 feet & 52.5 feet & 57 feet & 62 feet & 66.5 feet & 71 foet \\
\hline \multirow{2}{*}{$2 T+G=K$} & \multicolumn{6}{|c|}{$\begin{array}{c}\text { For intermediate values of } K \text { interpolate by using these } \\
\text { differences per unit }\end{array}$} \\
\hline & 82 & 98 & 115 & 136 & 156 & 178 \\
\hline $\begin{array}{l}68 \ldots \\
72 \ldots \\
76 \ldots \\
80 \ldots \\
84 \ldots\end{array}$ & $\begin{array}{r}\text { Lb.- }{ }^{-} t . \\
5,541 \\
5,867 \\
6,193 \\
6,519 \\
6,845\end{array}$ & $\begin{array}{r}\text { Lb. ft. } \\
7,019 \\
7,409 \\
7,799 \\
8,189\end{array}$ & $\begin{array}{c}\text { Lb.-ft. } \\
\\
9,193 \\
9,652\end{array}$ & $\begin{array}{r}L b .-f t . \\
10,880 \\
11,420\end{array}$ & Lb.-ft. & \begin{tabular}{l} 
Lb.-ft. \\
\hdashline-1 \\
\hdashline-1
\end{tabular} \\
\hline $\begin{array}{l}88 \\
92- \\
96 \\
100 \\
104\end{array}$ & $\begin{array}{l}7,171 \\
7,497 \\
7,823 \\
8,149 \\
8,475\end{array}$ & $\begin{array}{r}8,578 \\
8,968 \\
9,358 \\
9,748 \\
10,140\end{array}$ & $\begin{array}{l}10,110 \\
10,570 \\
11,030 \\
11,490 \\
11,950\end{array}$ & $\begin{array}{l}11,960 \\
12,510 \\
13,050 \\
13,600 \\
14,140\end{array}$ & $\begin{array}{l}13,760 \\
14,390 \\
15,010 \\
15,640 \\
16,270\end{array}$ & $\begin{array}{l}15,690 \\
16,400 \\
17,120 \\
17,830 \\
18,540\end{array}$ \\
\hline $\begin{array}{l}108 \\
112- \\
116- \\
120 \\
124\end{array}$ & $\begin{array}{r}8,801 \\
9,127 \\
9,453 \\
9,778 \\
10,100\end{array}$ & $\begin{array}{l}10,530 \\
10,920 \\
11,310 \\
11,700 \\
12,090\end{array}$ & $\begin{array}{l}12,410 \\
12,870 \\
13,330 \\
13,790 \\
14,250\end{array}$ & $\begin{array}{l}14,680 \\
15,230 \\
15,770 \\
16,310 \\
16,860\end{array}$ & $\begin{array}{l}16,890 \\
17,520 \\
18,140 \\
18,770 \\
19,390\end{array}$ & $\begin{array}{l}19,260 \\
19,970 \\
20,680 \\
21,390 \\
22,110\end{array}$ \\
\hline $\begin{array}{l}128 \\
132- \\
136- \\
140- \\
144_{--}\end{array}$ & $\begin{array}{l}10,430 \\
10,760 \\
11,080 \\
11,410 \\
11,730\end{array}$ & $\begin{array}{l}12,480 \\
12,870 \\
13,260 \\
13,650 \\
14,040\end{array}$ & $\begin{array}{l}14,710 \\
15,170 \\
15,630 \\
16,090 \\
16,550\end{array}$ & $\begin{array}{l}17,400 \\
17,950 \\
18,490 \\
19,030 \\
19,580\end{array}$ & $\begin{array}{l}20,020 \\
20,650 \\
21,270 \\
21,900 \\
22,520\end{array}$ & $\begin{array}{l}22,820 \\
23,530 \\
24,250 \\
24,960 \\
25,670\end{array}$ \\
\hline $\begin{array}{l}148 \\
152 \\
156 \\
160\end{array}$ & $\begin{array}{l}12,060 \\
12,390 \\
12,710 \\
13,040\end{array}$ & $\begin{array}{l}14,430 \\
14,820 \\
15,210 \\
15,600\end{array}$ & $\begin{array}{l}17,010 \\
17,470 \\
17,930 \\
18,390\end{array}$ & $\begin{array}{l}20,120 \\
20,660 \\
21,210 \\
21,750\end{array}$ & $\begin{array}{l}23,150 \\
23,770 \\
24,400 \\
25,020\end{array}$ & $\begin{array}{l}26,390 \\
27,100 \\
27,810 \\
28,530\end{array}$ \\
\hline $\begin{array}{l}164 \\
168 \\
172 \\
176\end{array}$ & $\begin{array}{l}13,360 \\
13,690 \\
14,020 \\
14,340\end{array}$ & $\begin{array}{l}15,990 \\
16,380 \\
16,770 \\
17,160\end{array}$ & $\begin{array}{l}18,850 \\
19,300 \\
19,760 \\
20,220\end{array}$ & $\begin{array}{l}22,300 \\
22,840 \\
23,380 \\
23,930\end{array}$ & $\begin{array}{l}25,650 \\
26,280 \\
26,900 \\
27,530\end{array}$ & $\begin{array}{l}29,240 \\
29,950 \\
30,670 \\
31,380\end{array}$ \\
\hline
\end{tabular}


Table 87B.--Moments due to wind pressure on poles-Continued LIGHT LOADING

\begin{tabular}{|c|c|c|c|c|c|c|c|c|}
\hline \multirow{2}{*}{$\begin{array}{c}T=\text { Top circum- } \\
\text { ference in } \\
\text { inches } \\
G=\text { Ground-line } \\
\text { circumference in } \\
\text { inches }\end{array}$} & \multicolumn{8}{|c|}{ Moment due to wind pressure on poles of various heights above ground } \\
\hline & 15 feet & 17 leet & 20 feet & 24.5 feet & 29 feet & 34 feet & 38.5 feet & 43 feet \\
\hline \multirow{2}{*}{$2 T+G=K$} & \multicolumn{8}{|c|}{ For intermediate values of $K$ interpolate by using these differences per unit } \\
\hline & 12 & 15 & 21 & 32 & 45 & 61 & 79 & 98 \\
\hline 84 & $\begin{array}{r}L b .-f t . \\
620 \\
668 \\
716 \\
764 \\
811\end{array}$ & $\begin{array}{r}L b .-f t . \\
797 \\
859 \\
920 \\
981 \\
1,043\end{array}$ & $\begin{array}{r}\text { Lb. }-f t . \\
1,103 \\
1,188 \\
1,273 \\
1,358 \\
1,443\end{array}$ & $\begin{array}{r}\text { Lb.-ft. } \\
1,783 \\
1,911 \\
2,038 \\
2,165\end{array}$ & $\begin{array}{r}L b .-f t . \\
2,499 \\
2,677 \\
2,855 \\
3,034\end{array}$ & $\begin{array}{r}L b .-f t . \\
3,680 \\
3,925 \\
4,170\end{array}$ & $\begin{array}{r}\text { Lb.-ft. } \\
4,718 \\
5,033 \\
5,347\end{array}$ & $\begin{array}{r}L b .-f t . \\
5,886 \\
6,278 \\
6,670\end{array}$ \\
\hline $\begin{array}{l}72 .- \\
76- \\
80 .- \\
84 .- \\
88 .\end{array}$ & $\begin{array}{r}859 \\
907 \\
955 \\
1,002 \\
1,050\end{array}$ & $\begin{array}{l}1,104 \\
1,165 \\
1,227 \\
1,288 \\
1,349\end{array}$ & $\begin{array}{l}1,528 \\
1,613 \\
1,698 \\
1,783 \\
1,867\end{array}$ & $\begin{array}{l}2,293 \\
2,420 \\
2,548 \\
2,675 \\
2,802\end{array}$ & $\begin{array}{l}3,212 \\
3,391 \\
3,569 \\
3,748 \\
3,926\end{array}$ & $\begin{array}{l}4,416 \\
4,661 \\
4,906 \\
5,152 \\
5,397\end{array}$ & $\begin{array}{l}5,662 \\
5,976 \\
6,291 \\
6,605 \\
6,920\end{array}$ & $\begin{array}{l}7,063 \\
7,455 \\
7,847 \\
8,240 \\
8,632\end{array}$ \\
\hline $\begin{array}{l}92 \ldots \\
96-. \\
100 \\
104- \\
108 .\end{array}$ & $\begin{array}{l}1,098 \\
1,146 \\
1,193 \\
1,241 \\
1,289\end{array}$ & $\begin{array}{l}1,411 \\
1,472 \\
1,533 \\
1,595 \\
1,656\end{array}$ & $\begin{array}{l}1,952 \\
2,037 \\
2,122 \\
2,207 \\
2,292\end{array}$ & $\begin{array}{l}2,930 \\
3,057 \\
3,184 \\
3,312 \\
3,439\end{array}$ & $\begin{array}{l}4,105 \\
4,283 \\
4,462 \\
4,640 \\
4,819\end{array}$ & $\begin{array}{l}5,642 \\
5,887 \\
6,133 \\
6,378 \\
6,623\end{array}$ & $\begin{array}{l}7,234 \\
7,549 \\
7,864 \\
8,178 \\
8,493\end{array}$ & $\begin{array}{r}9,025 \\
9,417 \\
9,809 \\
10,200 \\
10,590\end{array}$ \\
\hline $\begin{array}{l}112 \ldots \\
116 \ldots \\
120 \ldots \\
124 \ldots \\
128 \ldots\end{array}$ & $\begin{array}{r}1,337 \\
\end{array}$ & $\begin{array}{l}1,717 \\
1,779 \\
1,840\end{array}$ & $\begin{array}{l}2,377 \\
2,462 \\
2,546 \\
2,631 \\
\ldots . . .\end{array}$ & $\begin{array}{l}3,567 \\
3,694 \\
3,821 \\
3,949 \\
4,076\end{array}$ & $\begin{array}{l}4,997 \\
5,176 \\
5,354 \\
5,532 \\
5,711\end{array}$ & $\begin{array}{l}6,869 \\
7,114 \\
7,359 \\
7,605 \\
7,850\end{array}$ & $\begin{array}{r}8,807 \\
9,122 \\
9,436 \\
9,751 \\
10,070\end{array}$ & $\begin{array}{l}10,990 \\
11,380 \\
11,770 \\
12,160 \\
12,560\end{array}$ \\
\hline $\begin{array}{l}132 . \\
136 . \\
140 . \\
148 .\end{array}$ & & & & $\begin{array}{r}4,203 \\
\end{array}$ & $\begin{array}{r}5,889 \\
6,068 \\
6,246 \\
-\ldots . .\end{array}$ & $\begin{array}{l}8,095 \\
8,341 \\
8,586 \\
8,831 \\
9,076\end{array}$ & $\begin{array}{l}10,380 \\
10,690 \\
11,010 \\
11,320 \\
11,640\end{array}$ & $\begin{array}{l}12,950 \\
13,340 \\
13,730 \\
14,130 \\
14,520\end{array}$ \\
\hline $\begin{array}{l}152 .- \\
156=- \\
160- \\
164--\end{array}$ & & & & & & $\begin{array}{r}9,322 \\
9,567 \\
9,812 \\
10,060 \\
10,300\end{array}$ & $\begin{array}{l}11,950 \\
12,270 \\
12,580 \\
12,900 \\
13,210\end{array}$ & $\begin{array}{l}14,910 \\
15,300 \\
15,700 \\
16,090 \\
16,480\end{array}$ \\
\hline
\end{tabular}


Table 87B.-Moments due to wind pressure on poles-Continued LIGHT LOADING-Continued

\begin{tabular}{|c|c|c|c|c|c|c|}
\hline \multirow{2}{*}{$\begin{array}{l}T=T \text { op circumference in } \\
\text { inches } \\
G=\mathrm{Gr} \text { o und-line circumier- } \\
\text { ence in inches }\end{array}$} & \multicolumn{6}{|c|}{$\begin{array}{l}\text { Moment due to wind pressure on poles of various heights } \\
\text { above ground }\end{array}$} \\
\hline & 48 feet & 52.5 feet & 57 feet & 62 feet & 66.5 leet & 71 feet \\
\hline \multirow{2}{*}{$2 T+G=K$} & \multicolumn{6}{|c|}{$\begin{array}{c}\text { For intermediate values of } K \text { interpolate by using these differ } \\
\text { ences per unit }\end{array}$} \\
\hline & 122 & 145 & 172 & 205 & 235 & 270 \\
\hline $\begin{array}{l}68 .- \\
72- \\
76- \\
80- \\
84-\end{array}$ & $\begin{array}{r}L b .-f t . \\
8,312 \\
8,801 \\
9,290 \\
9,778 \\
10,270\end{array}$ & $\begin{array}{l}\text { Lo.-ft. } \\
10,530 \\
11,110 \\
11,700 \\
12,280\end{array}$ & Lb.-ft. & $\begin{array}{l}16,310 \\
17,130\end{array}$ & $L b .-f t$. & Lb.-ft. \\
\hline $\begin{array}{l}88 \ldots \\
92 \\
96 \\
100 \\
104\end{array}$ & $\begin{array}{l}10,760 \\
11,250 \\
11,730 \\
12,220 \\
12,710\end{array}$ & $\begin{array}{l}12,870 \\
13,450 \\
14,040 \\
14,620 \\
15,210\end{array}$ & $\begin{array}{l}15,170 \\
15,860 \\
16,550 \\
17,240 \\
17,930\end{array}$ & $\begin{array}{l}17,950 \\
18,760 \\
19,580 \\
20,390 \\
21,210\end{array}$ & $\begin{array}{l}20,650 \\
21,580 \\
22,520 \\
23,460 \\
24,400\end{array}$ & $\begin{array}{l}23,530 \\
24,600 \\
25,670 \\
26,740 \\
27,810\end{array}$ \\
\hline $\begin{array}{l}108 \\
112 \ldots \\
116 \ldots \\
120 \\
124\end{array}$ & $\begin{array}{l}13,200 \\
13,690 \\
14,180 \\
14,670 \\
15,160\end{array}$ & $\begin{array}{l}15,750 \\
16,380 \\
16,960 \\
17,550 \\
18,130\end{array}$ & $\begin{array}{l}18,620 \\
19,300 \\
19,990 \\
20,680 \\
21,370\end{array}$ & $\begin{array}{l}22,020 \\
22,840 \\
23,660 \\
24,470 \\
25,290\end{array}$ & $\begin{array}{l}25,340 \\
26,280 \\
27,210 \\
28,150 \\
29,090\end{array}$ & $\begin{array}{l}28,880 \\
29,950 \\
31,020 \\
32,090 \\
33,160\end{array}$ \\
\hline $\begin{array}{l}128 \\
132 \\
136 \\
140 \\
144\end{array}$ & $\begin{array}{l}15,650 \\
16,130 \\
16,620 \\
17,110 \\
17,600\end{array}$ & $\begin{array}{l}18,720 \\
19,309 \\
19,890 \\
20,470 \\
21,060\end{array}$ & $\begin{array}{l}22,060 \\
22,750 \\
23,440 \\
24,130 \\
24,820\end{array}$ & $\begin{array}{l}26,100 \\
26,920 \\
27,730 \\
28,550 \\
29,370\end{array}$ & $\begin{array}{l}30,030 \\
30,970 \\
31,910 \\
32,850 \\
33,780\end{array}$ & $\begin{array}{l}34,230 \\
35,300 \\
36,370 \\
37,440 \\
38,510\end{array}$ \\
\hline $\begin{array}{l}148 . \\
152 . \\
156 . \\
160 \ldots\end{array}$ & $\begin{array}{l}18,090 \\
18,580 \\
19,070 \\
19,560\end{array}$ & $\begin{array}{l}21,640 \\
22,230 \\
22,810 \\
23,400\end{array}$ & $\begin{array}{l}25,510 \\
26,200 \\
26,890 \\
27,580\end{array}$ & $\begin{array}{l}30,180 \\
31,000 \\
31,810 \\
32,630\end{array}$ & $\begin{array}{l}34,720 \\
35,660 \\
36,600 \\
37,540\end{array}$ & $\begin{array}{l}39,580 \\
40,650 \\
41,720 \\
42,790\end{array}$ \\
\hline $\begin{array}{l}164 \\
168 \\
172 \\
176\end{array}$ & $\begin{array}{l}20,050 \\
20,530 \\
21,020 \\
21,510\end{array}$ & $\begin{array}{l}23,980 \\
24,570 \\
25,150 \\
25,740\end{array}$ & $\begin{array}{l}28,270 \\
28,960 \\
29,650 \\
30,340\end{array}$ & $\begin{array}{l}33,440 \\
34,260 \\
35,080 \\
35,890\end{array}$ & $\begin{array}{l}38,480 \\
39,410 \\
40,350 \\
41,290\end{array}$ & $\begin{array}{l}43,860 \\
44,930 \\
46,000 \\
47,070\end{array}$ \\
\hline
\end{tabular}

The values of moments due to wind pressure given in Table $87 \mathrm{~B}$ are based upon a number $K$ which is found by adding the ground-line circumference to twice the value of the pole-top circumference in inches. By combining the two circumferences in this way, the table is simplified and greatly reduced in extent. Having found $K$, the value for the 
moment due to wind pressure is given in the same line as the value for $K$ and in the column which is headed by the height above ground of the given pole. For intermediate values of $K$ the proper value can be found by interpolation, using the differences given at the head of each column. The tabulated values give more significant figures than would otherwise be warranted, in order to avoid inaccuracies in interpolation.

Another method of determining the moment due to wind pressure on the pole is given in the nomogram of Figure 1. To use this nomogram, one determines, as before, the value of $K$, and this can be done graphically by laying a straightedge between proper points on the scales labeled $T$ and $G$. On scale $T$ find the value representing the circumference of the top of the pole in inches. On scale $G$ find the value for the ground-line circumference. The straight line joining these two points will intersect the scale for $K$ at a point giving its value. Now find on the scale of pole heights the proper point for a given pole and connect this point with the point already found on the $K$ scale. Where this straight line intersects the scale of moments will be found the desired value for the moment due to wind pressure on the given pole, in heavy or medium loading territory. For light loading territory, 50 per cent must be added to this value, since the loading specification for light loading territory is based upon a wind pressure of $12 \mathrm{lbs} . / \mathrm{ft} .^{2}$ whereas in heavy and medium territory the wind pressure is assumed to be only $8 \mathrm{lbs} . / \mathrm{ft}^{2}$ No consideration is given to a coating of ice in computing the wind pressure or bending moment on the pole.

When computing the total moment to be resisted by a pole under heavy or medium loading, supporting 10 wires or less, not including cables carried by messengers, it is found by adding together the moments due to each conductor and each cable and messenger and that due to the pole itself.

When the pole is carrying more than 10 wires not including cables supported by messengers, and the pin spacing does not exceed 15 inches, the moment due to the wires is calculated for two-thirds of the total number, but for not less than 10 wires. The moment due to the wires thus calculated is added to that due to the cables and messengers and the pole 


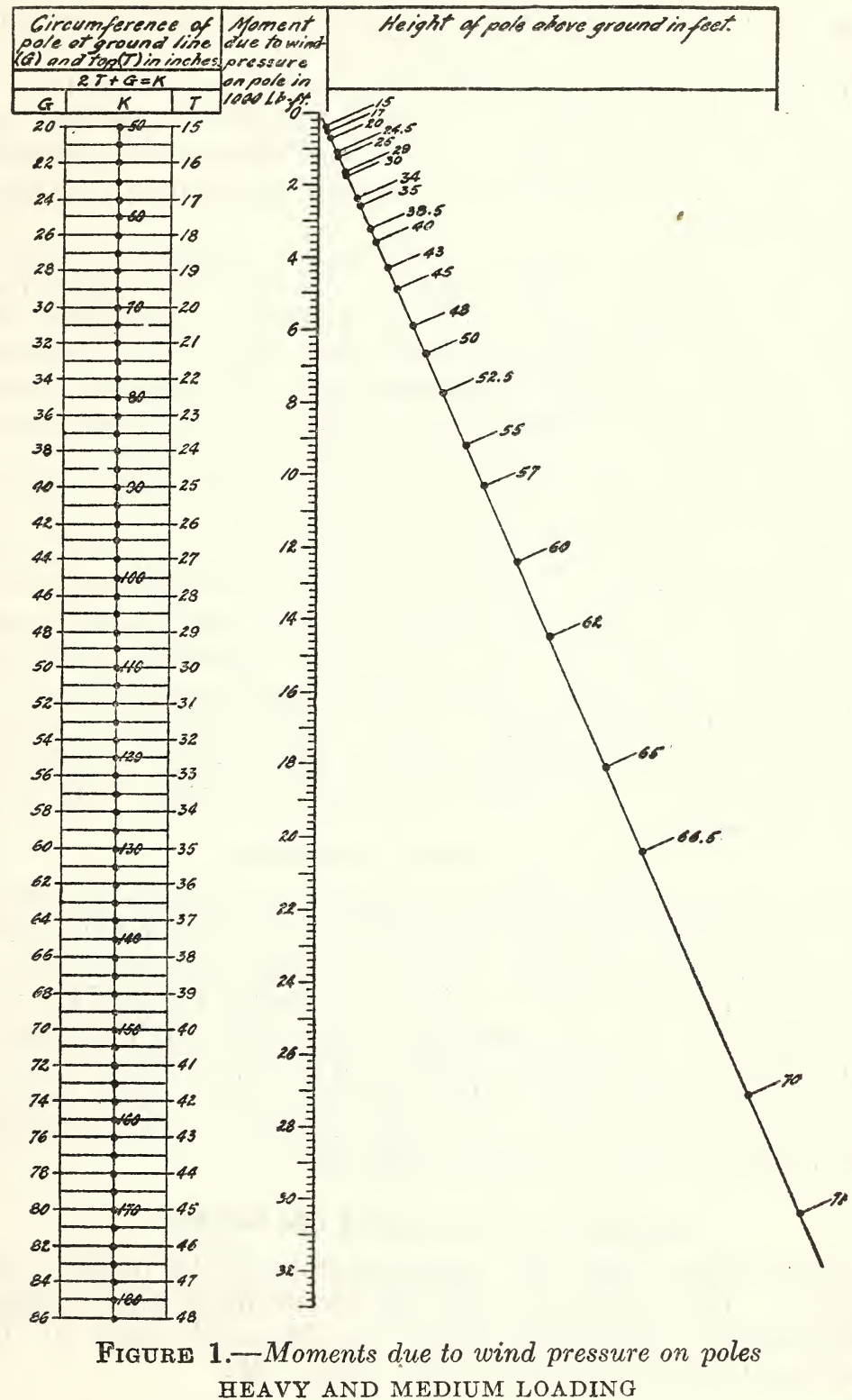

(For light loading, increase moments by 50 per cent) 
itself to find the total moment to be resisted by the pole under heavy or medium loading.

When computing the total moment to be resisted by a pole under light loading, it is found by adding together the moment due to each wire or cable and that due to the pole itself with no mitigation even though the number of wires carried is large.

When a trolley contact conductor is supported from a pole also carrying other wires or cables, the transverse moment resulting from the trolley contact conductors is added as part of the total moment to be resisted by the pole.

At angles in a line an unbalanced side pull is imposed on the corner pole due to change in direction of the conductors. The total moment to be resisted in this case is that due to a transverse load equal to the resultant of all conductor and messenger tensions under loaded conditions.

When considering the safety of poles of grades A, B, C, D, or $\mathrm{E}$, the fiber stress caused by the moments of the transverse wind forces on poles and conductors must be added to the fiber stresses caused by the vertical loads carried by the pole. The sum of these fiber stresses should be less than the maximum allowable fiber stress. Table 20 lists maximum allowable fiber stresses for certain timbers, and Table 87A gives the factors of safety for different situations of grade A, B, or C construction.

\section{VERTICAL LOADS ON POLES}

The vertical force acting on conductors in lines of grades $\mathrm{A}$, $\mathrm{B}, \mathrm{C}, \mathrm{D}$, or E for any loading district may be found by use of the values of the weight of loaded conductors per foot of length given in the National Electrical Safety Code, Table 81. The vertical force in pounds for each conductor is obtained by multiplying the loaded weight per foot by the span in feet or by one-half the sum of the adjacent spans. The vertical force supported by the pole is the sum of conductor loads. The weight of the pole itself is neglected.

\section{LONGITUDINAL LOADS ON POLES}

Longitudinal loads on a pole result from the pulls of conductors. The conductor pulls are determined under loaded conditions in conformity with the assumed loads of the National Electrical Safety Code, section 25. 
The bending moment in pound-feet at the ground line is determined by multiplying the pull in pounds of each conductor contributing to the longitudinal load under the loading assumptions by its height in feet. If the conductors are strung to the recommended sags of Appendix $A$, the pull in the conductors under loaded conditions may be found in Tables 38 to 43, inclusive, of Appendix A, National Electrical Safety Code. These tables cover various types of copper conductors. If the conductors are strung to the minimum permissible sags of grades $\mathrm{A}, \mathrm{B}$, or $\mathrm{C}$, the tensions will be 50 per cent of the breaking tension for grades $A$ and $B$ and 60 per cent for grade C. The breaking tensions of various types of line conductors may be found in the tables of mechanical data for wires and cables of Appendix D, National Electrical Safety Code. If the conductors are strung to sags differing from those mentioned above, the tensions under loaded conditions may be obtained by calculation.

The longitudinal pull of communication conductors may always be assumed to be one-half their breaking tension regardless of the sag (see N. E. S. C., Appendix D, for tables of breaking strength), except in grade A or B joint lines, where this is applicable only if conductors are smaller than No. 8 Stl. W. G. steel or No. 6 A. W. G. copper.

At dead ends and angles the longitudinal moment is computed by adding together the moments of all unbalanced conductor and cable pulls. In these situations the resisting moment of the pole is based on the allowable maximum fiber stresses for transverse loads in the same loading district and of the same grade of construction. In all other situations the resisting moment of the pole is based on the allowable fiber stresses for longitudinal loads.

When computing the longitudinal moment where a change of grade occurs in a line, the longitudinal loading upon poles at the ends of sections required to be grade $\mathrm{A}$ or $\mathrm{B}$ construction in lines of a lower grade is taken as an unbalanced pull in the direction of the higher-grade section equal to the total pull in one direction of all conductors and cables supported. This longitudinal load may be mitigated for higher-grade sections having no span exceeding 500 feet in length where the total pull in the direction of the higher grade exceeds 30,000 pounds. 
In this case the longitudinal force is modified to 30,000 pounds plus one-fourth the excess above 30,000 , with a maximum of 50,000 pounds.

When computing the longitudinal moment where a line is built throughout its length, or between dead-ended points, of grade A or B construction, although not so required, the longitudinal force upon poles at crossings, at ends of sections of joint use, and at ends of conflicts required to be of grade A or B construction, respectively, is taken as an unbalanced pull in the direction of the crossing, conflict or joint-use section equal to the pull of one-third of the total number of conductors carried, not including overhead ground wires. The one-third selected for computation must be a whole number or the next larger whole number and must be selected to produce the maximum stress in the pole.

\section{DEPRECIATION OF WOOD POLES}

The original Tables 86 and 88 in the National Electrical Safety Code depend upon the ratio of fiber stress when installed to fiber stress when depreciated and consequently are not affected by the absolute value of the ultimate fiber stress. These tables consequently remain unchanged and it is not considered necessary to reproduce them here.

\section{ALLOWABLE NUMBER OF WIRES ON A GIVEN POLE WITHOUT SIDE GUYS}

Table 89 in the National Electrical Safety Code gives the allowable number of No. 4 wires to be carried by 35 -foot poles of woods having an ultimate fiber stress of 5,000 lbs./in. ${ }^{2}$ This table will no longer apply to chestnut and western red cedar, which under the new standards are permitted to carry a larger load.

Tables 90 and 91 applying to poles which are supported by side guys are not affected. 

